\title{
HIGHER ORDER DERIVATIVE ESTIMATES FOR FINITE-DIFFERENCE SCHEMES FOR LINEAR ELLIPTIC AND PARABOLIC EQUATIONS*
}

\author{
ISTVÁN GYÖNGY† AND NICOLAI KRYLOV
}

\begin{abstract}
We give sufficient conditions under which solutions of finite-difference schemes in the space variable for second order possibly degenerate linear parabolic and elliptic equations admit estimates of spatial derivatives up to any given order independent of the mesh size.
\end{abstract}

Key words. Finite-difference approximations, linear elliptic and parabolic equations.

AMS subject classifications. 65M15, 35J70, 35K65

1. Introduction. This is the second part of a series of papers devoted to studying the smoothness of solutions to finite difference schemes for parabolic and elliptic partial differential equations given on the whole $\mathbb{R}^{d}$. These equations can degenerate, for example be just first order PDEs. As in [14], the first part of this series, we consider a grid in $\mathbb{R}^{d}$ and a large class of monotone finite difference schemes in the space variable $x$ in $\mathbb{R}^{d}$.

For each small parameter $h>0$ the given grid is dilated by $h$ and for each $x \in \mathbb{R}^{d}$ it is shifted so that $x$ becomes a mesh point. We are interested in the smoothness in $x$ of the solution $u_{h}(t, x)$ of the difference scheme. In [14] estimates, independent of $h$, for the first order derivatives of $u_{h}$ in $x$ were obtained under general conditions introduced there. In the present paper we investigate the higher order derivatives of $u_{h}$ in $x$. The main results give estimates, independent of $h$, for the derivatives of $u_{h}$ in $x$ up to any given order $m$. The conditions extend those from [14]. Using these results in the continuation of this paper we estimate the derivatives of $u_{h}$ in $h$, and that allows us to develop a new method of obtaining the power series of $u_{h}$ in $h$. Hence we get accelerated finite-difference schemes by using Richardson's extrapolation. Namely, under general conditions we show that the accuracy of finite difference schemes for parabolic and elliptic PDEs can be improved to any order by taking suitable linear combinations of finite difference approximations with different mesh-sizes. For elliptic PDEs this result is announced by Theorem 2.4 in [14] and for parabolic PDEs by Theorem 2.3 below. We hope to develop these results in domains for uniformly nondegenerate equations later.

Derivative estimates for finite-difference approximations for linear and for nonlinear PDEs play the paramount role in establishing the rate of convergence of the approximations. The importance of such estimates is demonstrated recently by [20], [21] and [22], presenting the first rate of convergence result in the sup norm of finitedifference approximations for fully nonlinear degenerate Bellman equations. Ideas from these publications are used and developed further in [2], [17], [3], [7], [8], [9] and [16]. Recent results on estimating the Lipschitz constant and second order differences of finite-difference approximations for a large class of fully nonlinear degenerate

\footnotetext{
*Received November 30, 2008; accepted for publication March 25, 2009.

${ }^{\dagger}$ School of Mathematics, University of Edinburgh, King's Buildings, Edinburgh, EH9 3JZ, United Kingdom (gyongy@maths.ed.ac.uk).

${ }^{\ddagger}$ School of Mathematics, 127 Vincent Hall, University of Minnesota, Minneapolis, MN 55455, USA (krylov@math.umn.edu). The work of the second author was partially supported by NSF grant DMS-0653121.
} 
PDEs, including the normalized Bellman equations are presented in [24]. In [8] first order derivatives of finite-difference approximations to degenerate linear parabolic and elliptic PDEs are estimated and are used to establish sharp estimates on the rate of convergence of the approximations in the sup norm.

Finite-difference methods for solving PDEs have been extensively studied since the first half of the last century. Let us mention the pioneering papers by R. Courant, K.O. Friedrichs and H. Lewy [6], S. Gerschgorin [12], and publications by D.G. Aronson, J. Douglas, F. John, H.O. Kreiss, O. Ladyzhenskaya, P.D. Lax, W. Littman, Lyusternik, J. von Neumann, I.G. Petrovskii, A.A. Samarskii, G. Strang, A.N. Tikhonov, V. Thomée, O.B. Widlund and many others (see, e.g., [1], [10], [18], [19], [27], [28], [29], [30], [32], [33], [36], [40], [41] and the references there.) We refer also to the review paper [38], handbook [39], and well-known monographs and textbooks for more information on the subject ([5], [11], [13], [26], [31], [34], [35], [37]).

The paper is organized as follows. The main results, Theorems 2.1 and 2.2 are presented in Section 2. Here we formulate also a result, Theorem 2.3, on accelerated finite difference schemes, which we will prove in the continuation of this paper by using Theorem 2.1. As we have pointed out above, the idea of the proof of Theorem 2.3 is based on a power expansion of $u_{h}$ in $h$. This idea was already applied by the authors to show how to accelerate other approximation schemes (see, for instance, [15]) and it seems to the authors that it was never used before in the framework of finite difference schemes in the sup norm for degenerate elliptic and parabolic equations although much effort was applied to developing this and other methods of improved approximation for uniformly nondegenerate equations in domains (see, for instance [4] and the references therein). It is worth saying that, in contrast with [4] and many other papers dealing with the expansion, we do not use any information from the theory of PDE and, as a matter of fact, the existence of smooth solutions for degenerate elliptic and parabolic equations follows directly from our results. We deduce Theorem 2.2 from Theorem 2.1 , and conclude Section 2 with verifying the rather delicate conditions of the main results, Assumptions 2.4 and 2.5, for a class of examples, given in Remark 2.2 before the formulation of the theorems. The proof of Theorem 2.1 is given in Section 3, and the final section, Section 4, is devoted to further discussions of Assumptions 2.4 and 2.5 .

The authors are sincerely grateful to the referees for many useful comments and suggestions.

2. Formulation of the main results. We take some numbers $h_{0}, T \in(0, \infty)$ and for each number $h \in\left(0, h_{0}\right]$ we consider the integral equation

$$
u(t, x)=g_{h}(x)+\int_{0}^{t}\left(L_{h} u(s, x)+f_{h}(s, x)\right) d s, \quad(t, x) \in H_{T}
$$

for $u$, where $g=g_{h}=g_{h}(x)$ and $f=f_{h}=f_{h}(s, x)$ are given real-valued Borel functions of $x \in \mathbb{R}^{d}$ and $(s, x) \in H_{T}=[0, T] \times \mathbb{R}^{d}$, respectively, and $L=L_{h}$ is a linear operator defined by

$$
\begin{gathered}
L_{h} \varphi(t, x)=L_{h}^{0} \varphi(t, x)-c(t, x) \varphi(x), \\
L^{0} \varphi(t, x)=L_{h}^{0} \varphi(t, x)=\frac{1}{h} \sum_{\lambda \in \Lambda_{1}} q_{\lambda}(t, x) \delta_{h, \lambda} \varphi(x)+\sum_{\lambda \in \Lambda_{1}} p_{\lambda}(t, x) \delta_{h, \lambda} \varphi(x),
\end{gathered}
$$


for functions $\varphi$ on $\mathbb{R}^{d}$. Here $\Lambda_{1}$ is a finite subset of $\mathbb{R}^{d}$ such that $0 \notin \Lambda_{1}$, and $p_{\lambda}(t, x)$, $q_{\lambda}(t, x)$ are real-valued functions of $(t, x) \in H_{\infty}=[0, \infty) \times \mathbb{R}^{d}$ given for each $\lambda \in \Lambda_{1}$, and

$$
\delta_{\lambda} \varphi(x)=\delta_{h, \lambda} \varphi(x)=\frac{1}{h}(\varphi(x+h \lambda)-\varphi(x)), \quad \lambda \in \Lambda_{1} .
$$

As usual, for multi-indices $\alpha=\left(\alpha_{1}, \ldots \alpha_{d}\right), \alpha_{i}=0,1, \ldots$, we use the notation

$$
D^{\alpha}=D_{1}^{\alpha_{1}} \ldots D_{d}^{\alpha_{d}}, \quad D_{i}=\frac{\partial}{\partial x_{i}}, \quad|\alpha|=\sum_{i} \alpha_{i}, \quad D_{i j}=D_{i} D_{j} .
$$

For smooth $\varphi$ and integers $k \geq 0$ we introduce $D^{k} \varphi$ as the collection of partial derivatives of $\varphi$ of order $k$, and define

$$
\left|D^{k} \varphi\right|^{2}=\sum_{|\alpha|=k}\left|D^{\alpha} \varphi\right|^{2}, \quad[\varphi]_{k}=\sup _{x \in \mathbb{R}^{d}}\left|D^{k} \varphi(x)\right|, \quad|\varphi|_{k}=\sum_{i \leq k}[\varphi]_{i} .
$$

Let $m \geq 0$ be a fixed integer and let $K_{1} \in[1, \infty)$ be a constant. We make the following assumptions.

Assumption 2.1. For any $\lambda \in \Lambda_{1}$ the derivatives in $x$ of $p_{\lambda}, q_{\lambda}, c, f, g$ up to order $m$ are continuous functions in $(t, x) \in H_{T}$ and, for $k=0, \ldots, m$ and some constants $M_{k}$ we have

$$
\sup _{H_{T}}\left(\sum_{\lambda \in \Lambda_{1}}\left(\left|D^{k} q_{\lambda}\right|^{2}+\left|D^{k} p_{\lambda}\right|^{2}\right)+\left|D^{k} c\right|^{2}\right) \leq M_{k}^{2}
$$

By Theorem 2.3 of [14] under Assumption 2.1 for each $h \in\left(0, h_{0}\right]$, there exists a unique bounded solution $u_{h}$ of (2.1) and this solution is continuous in $H_{T}$ along with all its derivatives in $x$ up to order $m$. However, the bounds, provided by this theorem for these derivatives depend on the parameter $h$. Our aim is to show the existence of bounds, independent of $h$, if in addition to Assumption 2.1, the assumptions below also hold.

Assumption 2.2. For all $t \in[0, T]$

$$
\sum_{\lambda \in \Lambda_{1}} \lambda q_{\lambda}(t, x) \quad \text { is independent of } x .
$$

This assumption may look to be a very restrictive condition. Note, however, that a simple application of Taylor's formula shows that if our finite difference operators $L_{h}^{0}$ appear as finite-difference approximations of a second-order differential operator, then not only the sum appearing in the assumption does not depend on $x$ but it is just identically zero. It is also worth noting that Assumption 2.2 is satisfied in many other cases, say if $q_{\lambda}(x)$ are just independent of $x$ or they are independent of $\lambda$ and $\sum_{\lambda \in \Lambda_{1}} \lambda=0$. The reader can find in [14] many more interesting cases when this and our other assumptions are satisfied.

Introduce

$$
\chi_{\lambda}=\chi_{h, \lambda}=q_{\lambda}+h p_{\lambda} .
$$


Assumption 2.3. For all $(t, x) \in H_{T}, h \in\left(0, h_{0}\right]$, and $\lambda \in \Lambda_{1}$,

$$
\chi_{\lambda}(t, x) \geq 0 .
$$

There exists a constant $c_{0}>0$ such that $c \geq c_{0}$.

Obviously Assumption 2.3 implies that $q_{\lambda} \geq 0$. Note also that Assumption 2.3 is a standard condition to have a monotone finite-difference approximation schemes to elliptic differential operators.

REMARK 2.1. The above assumption: $c \geq c_{0}>0$, is almost irrelevant if we only consider (2.1) on a finite time interval. Indeed, if $c$ is just bounded, say $|c| \leq$ $C=$ const, by introducing a new function $v(t, x)=u(t, x) e^{-2 C t}$ we will have an equation for $v$ similar to (2.1) with $L^{0} v-(c+2 C) v$ and $f e^{-2 C t}$ in place of $L u$ and $f$, respectively. Now for the new $c$ we have $c+2 C \geq C$.

Take a function $\tau_{\lambda}$ defined on $\Lambda_{1}$ taking values in $[0, \infty)$ and for $\lambda \in \Lambda_{1}$ introduce the operators

$$
T_{\lambda} \varphi=T_{h, \lambda} \varphi(x)=\varphi(x+h \lambda), \quad \bar{\delta}_{\lambda}=\bar{\delta}_{h, \lambda}=\tau_{\lambda} h^{-1}\left(T_{\lambda}-1\right) .
$$

We introduce weights $\tau_{\lambda}$ in order to be able to prove such estimates of finite differences of solutions, which are applicable to estimating the finite differences with respect to a parameter entering the equation (see Remark 5.3 of [14]).

Apart from estimating finite differences of solutions we also estimate their derivatives and for uniformity of notation we also introduce $\Lambda_{2}$ as the set of fixed distinct vectors $\ell^{1}, \ldots, \ell^{d}$ none of which is in $\Lambda_{1}$ and define

$$
\bar{\delta}_{\ell^{i}}=\bar{\delta}_{h, \ell^{i}}=\tau_{0} D_{i}, \quad T_{\ell^{i}}=T_{h, \ell^{i}}=1, \quad \Lambda=\Lambda_{1} \cup \Lambda_{2},
$$

where $\tau_{0}$ is a fixed parameter satisfying

$$
\tau_{0}>0
$$

so that the derivatives of solutions will be always present in our estimates. For integers $k=1,2, \ldots$ and $\lambda^{i} \in \Lambda, i=1,2, \ldots, k$, introduce the multi-vectors

$$
\lambda=\left(\lambda^{1}, \ldots, \lambda^{k}\right) \in \Lambda^{k}
$$

and the operators

$$
T_{\lambda}=T_{h, \lambda}=T_{h, \lambda^{1}} \ldots T_{h, \lambda^{k}}, \quad \bar{\delta}_{\lambda}=\bar{\delta}_{h, \lambda}=\bar{\delta}_{h, \lambda^{1} \ldots} \bar{\delta}_{h, \lambda^{k}} .
$$

It is also convenient to set $\Lambda_{1}^{0}=\Lambda_{2}^{0}=\Lambda^{0}=\{0\}$ and to introduce $\delta_{0}=\bar{\delta}_{0}$ and $T_{0}$ as unit operators. For $\mu \in \Lambda^{k}$ and $k \leq m$ we set

$$
\begin{gathered}
Q \varphi=h^{-1} \sum_{\lambda \in \Lambda_{1}} q_{\lambda} \delta_{\lambda} \varphi, \quad Q_{\mu} \varphi=h^{-1} \sum_{\lambda \in \Lambda_{1}}\left(\bar{\delta}_{\mu} q_{\lambda}\right) \delta_{\lambda} \varphi \\
P \varphi=\sum_{\lambda \in \Lambda_{1}} p_{\lambda} \delta_{\lambda} \varphi, \quad P_{\mu} \varphi=\sum_{\lambda \in \Lambda_{1}}\left(\bar{\delta}_{\mu} p_{\lambda}\right) \delta_{\lambda} \varphi, \\
L_{\mu}^{0}=Q_{\mu}+P_{\mu},
\end{gathered}
$$




$$
A_{k}(\varphi)=2 \sum_{\lambda \in \Lambda^{k}}\left(\bar{\delta}_{\lambda} \varphi\right) L_{\lambda}^{0} T_{\lambda} \varphi, \quad \mathcal{Q}(\varphi)=\sum_{\mu \in \Lambda_{1}} \chi_{\mu}\left(\delta_{\mu} \varphi\right)^{2}
$$

Below $B\left(\mathbb{R}^{d}\right)$ is the set of bounded Borel functions on $\mathbb{R}^{d}$ and $\mathfrak{K}$ is the set of bounded operators $\mathcal{K}=\mathcal{K}_{h}=\mathcal{K}_{h}(t)$ mapping $B\left(\mathbb{R}^{d}\right)$ into itself preserving the cone of nonnegative functions and satisfying $\mathcal{K} 1 \leq 1$. Set

$$
\left|\Lambda_{1}\right|^{2}=\sum_{\lambda \in \Lambda_{1}}|\lambda|^{2}, \quad\left\|\Lambda_{1}\right\|^{2}=\sum_{\lambda \in \Lambda_{1}}\left|\tau_{\lambda} \lambda\right|^{2}
$$

Finally, fix a constant $\delta \in(0,1]$.

Assumption 2.4. We have $m \geq 1$ and for any $h \in\left(0, h_{0}\right]$, there exists an operator $\mathcal{K}=\mathcal{K}_{h, m} \in \mathfrak{K}$, such that

$$
m A_{1}(\varphi) \leq(1-\delta) \sum_{\lambda \in \Lambda} \mathcal{Q}\left(\bar{\delta}_{\lambda} \varphi\right)+K_{1} \mathcal{Q}(\varphi)+2(1-\delta) c \mathcal{K}\left(\sum_{\lambda \in \Lambda}\left|\bar{\delta}_{\lambda} \varphi\right|^{2}\right)
$$

on $H_{T}$ for all smooth functions $\varphi$.

Assumption 2.5. We have $m \geq 2$ and, for any $h \in\left(0, h_{0}\right]$ and $n=1, \ldots, m$, there exists an operator $\mathcal{K}=\mathcal{K}_{h, n} \in \mathfrak{K}$, such that

$$
\begin{aligned}
& n \sum_{\nu \in \Lambda} A_{1}\left(\bar{\delta}_{\nu} \varphi\right)+n(n-1) \sum_{\lambda \in \Lambda^{2}}\left(\bar{\delta}_{\lambda} \varphi\right) Q_{\lambda} T_{\lambda} \varphi \leq(1-\delta) \sum_{\lambda \in \Lambda^{2}} \mathcal{Q}\left(\bar{\delta}_{\lambda} \varphi\right) \\
& +K_{1} \sum_{\lambda \in \Lambda} \mathcal{Q}\left(\bar{\delta}_{\lambda} \varphi\right)+2(1-\delta) c \mathcal{K}\left(\sum_{\lambda \in \Lambda^{2}}\left|\bar{\delta}_{\lambda} \varphi\right|^{2}\right)+K_{1} \mathcal{K}\left(\sum_{\lambda \in \Lambda}\left|\bar{\delta}_{\lambda} \varphi\right|^{2}\right)
\end{aligned}
$$

on $H_{T}$ for all smooth functions $\varphi$.

Obviously Assumptions 2.4 and 2.5 are satisfied if $q_{\lambda}$ and $p_{\lambda}$ are independent of $x$. In the general case, as it is discussed in [14], the above assumptions impose not only analytical conditions, but they are related also to some structural conditions, which can somewhat easier be analyzed under the following symmetry condition:

(S) $\Lambda_{1}=-\Lambda_{1}$ and $q_{\lambda}=q_{-\lambda}$ for all $\lambda \in \Lambda_{1}$.

Notice that, if condition (S) holds then

$$
h^{-1} \sum_{\lambda \in \Lambda_{1}} q_{\lambda}(t, x) \delta_{\lambda} \varphi(x)=(1 / 2) \sum_{\lambda \in \Lambda_{1}} q_{\lambda}(t, x) \Delta_{\lambda} \varphi(x),
$$

where $\Delta_{\lambda}=\Delta_{h, \lambda}$ and

$$
\Delta_{h, \lambda} \varphi(x)=\frac{\varphi(x+h \lambda)-2 \varphi(x)+\varphi(x-h \lambda)}{h^{2}}=-\delta_{-\lambda} \delta_{\lambda} \varphi(x) .
$$

Notice also that (S) implies that the sum in (2.3) in Assumption 2.2 is identically zero since the sum changes sign if we replace $\lambda$ with $-\lambda$.

Remark 2.2. Assumption 2.4 is discussed at length and in many details in [14]. In this remark we suppose that Assumptions 2.1, 2.2, and 2.3 hold and $m \geq 2$. At the end of this section we show that if condition (S) holds and, for all $\lambda \in \Lambda_{1}, \tau_{\lambda}>0$ and $q_{\lambda} \geq \kappa$, where $\kappa>0$ is a constant, then both Assumptions 2.4 and 2.5 are satisfied for 
any $c_{0}>0$ and $\delta \in(0,1)$, if $h_{0}$ is sufficiently small and $\tau_{0}>0, K_{1}$, and $\mathcal{K}$ are chosen appropriately. In the case $\tau_{\lambda} \equiv 1$ it follows immediately from Remark 6.4 of [14] and Remark 4.3 that the above condition $\kappa>0$ can be dropped, provided, additionally, that $c_{0}$ is large enough (this time we need not assume that $h$ is small).

By the way, as we have seen in Remark 2.1, the condition that $c_{0}$ be large is, actually, harmless as long as we are concerned with equations on a finite time interval.

Mixed situations, when $c$ is large at those points where some of $q_{\lambda}$ can vanish are considered in Section 4 along with the analysis of Assumption 2.4 as $h \downarrow 0$.

Now we are in the position to formulate our main results. Recall that as we have pointed out after Assumption 2.1 all derivatives of $u_{h}$ with respect to $x$ up to order $m$ are continuous in $H_{T}$.

TheOREm 2.1. Let Assumptions 2.1 through 2.5 hold. Then for $h \in\left(0, h_{0}\right]$ we have

$$
\sup _{H_{T}} \sum_{k=0}^{m}\left|D^{k} u_{h}\right| \leq N\left(F_{m}+G_{m}\right) \text {, }
$$

where

$$
F_{n}=\sum_{k \leq n} \sup _{H_{T}}\left|D^{k} f_{h}\right|, \quad G_{n}=\sum_{k \leq n} \sup _{\mathbb{R}^{d}}\left|D^{k} g_{h}\right|
$$

and $N$ depends only on $\tau_{0}, m, \delta, c_{0}, K_{1},\left|\Lambda_{1}\right|,\left\|\Lambda_{1}\right\|, M_{0}, \ldots, M_{m}$.

We prove this theorem in Section 3. Now we derive from it an estimate for the solution of the equation

$$
L_{h} v+f_{h}=0 \quad \text { in } \mathbb{R}^{d}
$$

when $q_{\lambda}, p_{\lambda}, c$, and $f$ are independent of $t$.

Theorem 2.2. Let Assumptions 2.1 through 2.4 be satisfied. Suppose that $q_{\lambda}$, $p_{\lambda}, c$, and $f$ are independent of $t$. Then the following statements hold.

(i) There exists a unique bounded solution $v=v_{h}(x)$ of (2.8). Moreover, all derivatives in $x$ of $v$ up to order $m$ are bounded continuous functions on $\mathbb{R}^{d}$.

(ii) Let Assumption 2.5 be also satisfied. Then

$$
\sup _{\mathbb{R}^{d}} \sum_{k=0}^{m}\left|D^{k} v_{h}\right| \leq N F_{m}
$$

where

$$
F_{m}=\sum_{k=0}^{m} \sup _{\mathbb{R}^{d}}\left|D^{k} f_{h}\right|
$$

and $N$ depends only on $\tau_{0}, m, \delta, c_{0}, K_{1},\left|\Lambda_{1}\right|,\left\|\Lambda_{1}\right\|, M_{0}, \ldots, M_{m}$.

Proof. Statement (i) is proved in [14] (see Theorem 2.3 there). To prove (ii) take $\nu=c_{0} \gamma$, where $\gamma>0$ is so small that $c-\nu \geq c_{0} / 2$ and conditions (2.5) and (2.6) 
hold with $c-\nu$ and $\delta / 2$ in place of $c$ and $\delta$, respectively. Define $u(t, x):=v(x) e^{\nu t}$ and observe that $u$ satisfies

$$
\frac{\partial}{\partial t} u=L^{0} u-(c-\nu) u+e^{\nu t} f .
$$

By Theorem 2.1 for $x \in \mathbb{R}^{d}$

$$
e^{\nu T} \sum_{k=0}^{m}\left|D^{k} v(x)\right|=\sum_{k=0}^{m}\left|D^{k} u(T, x)\right| \leq N e^{\nu T} F_{m}+N \sum_{k \leq m} \sup _{\mathbb{R}^{d}}\left|D^{k} v(x)\right| .
$$

By multiplying the extreme terms by $e^{-\nu T}$ and letting $T \rightarrow \infty$, we get the result.

The above theorems have important applications in the numerical analysis of finite difference schemes for parabolic and elliptic PDEs. Using them in the continuation of the present paper we obtain accelerated finite difference schemes for second order (possibly) degenerate parabolic and also for second order (possibly) degenerate elliptic PDEs. In particular, we will consider the Cauchy problem

$$
\begin{gathered}
\frac{\partial}{\partial t} u(t, x)=\mathcal{L} u(t, x)+f_{0}(t, x), \quad t \in(0, T], x \in \mathbb{R}^{d} \\
u(0, x)=g_{0}(x), \quad x \in \mathbb{R}^{d}
\end{gathered}
$$

with the operator

$$
\mathcal{L}:=\frac{1}{2} \sum_{\lambda \in \Lambda_{1}} \sum_{i, j=1}^{d} q_{\lambda} \lambda_{i} \lambda_{j} D_{i} D_{j}+\sum_{\lambda \in \Lambda_{1}} \sum_{i=1}^{d} p_{\lambda} \lambda_{i} D_{i}-c .
$$

By a solution of (2.9)-(2.10) we mean a continuous function $u(t, x)$ on $H_{T}$, such that for each $t$ it is twice continuously differentiable in $x$, is bounded in $H_{T}$ along with its derivatives in $x$ up to second order and satisfies

$$
u(t, x)=g_{0}(x)+\int_{0}^{t}\left[\mathcal{L} u(s, x)+f_{0}(s, x)\right] d s
$$

in $H_{T}$.

To formulate one of the main results of the continuation of the paper we fix an integer $k \geq 0$ and set $f_{h}=f_{0}, g_{h}=g_{0}$,

$$
\bar{u}_{h}=\sum_{j=0}^{k} b_{j} u_{2^{-j} h}
$$

where $u_{2^{-j} h}$ is the solution to (2.1) with $2^{-j} h$ in place of $h$,

$$
\left(b_{0}, b_{1}, \ldots, b_{k}\right):=(1,0,0, \ldots, 0) V^{-1},
$$

and $V^{-1}$ is the inverse of the Vandermonde matrix with entries

$$
V^{i j}:=2^{-(i-1)(j-1)}, \quad i, j=1, \ldots, k+1 .
$$


TheOrem 2.3. Let Assumptions 2.3 and 2.1 with $m \geq 3(k+1)$ hold. Also let condition (S) be satisfied. Then (2.9)-(2.10) has a unique solution $u_{0}$, and

$$
\left|\bar{u}_{h}(t, x)-u_{0}(t, x)\right| \leq N h^{k+1}
$$

holds for all $(t, x) \in H_{T}, h \in\left(0, h_{0}\right]$, where $N$ is a constant depending only on $T, k$, $d,\left|\Lambda_{1}\right|, h_{0}$, the number of elements in $\Lambda_{1}$, on $M_{0}, \ldots M_{m}$, on $\sup _{t \in[0, T]}\left|f_{0}(t)\right|_{m}$ and on $\left|g_{0}\right|_{m}$.

Now we prove our claim made in Remark 2.2. Instead of condition (S) we assume the following weaker condition

$\left(\mathrm{S}^{\prime}\right): \Lambda_{1}=-\Lambda_{1}$ and $D q_{\lambda}=D q_{-\lambda}$ for $\lambda \in \Lambda_{1}$,

and proceed with the proof as follows. Clearly,

$$
\sum_{\lambda \in \Lambda}\left(\bar{\delta}_{\lambda} \varphi\right) L_{\lambda}^{0} T_{\lambda} \varphi=I_{1}+I_{2}
$$

with

$$
I_{1}:=\sum_{\lambda \in \Lambda_{1}}\left(\bar{\delta}_{\lambda} \varphi\right) L_{\lambda}^{0} T_{\lambda} \varphi, \quad I_{2}:=\sum_{\lambda \in \Lambda_{2}}\left(\bar{\delta}_{\lambda} \varphi\right) L_{\lambda}^{0} \varphi .
$$

Due to condition $\left(\mathrm{S}^{\prime}\right)$

$$
\begin{gathered}
I_{1}=\sum_{\lambda \in \Lambda_{1}}\left(\bar{\delta}_{\lambda} \varphi\right) L_{\lambda}^{0} \varphi+h \sum_{\lambda \in \Lambda_{1}}\left(\bar{\delta}_{\lambda} \varphi\right) L_{\lambda}^{0} \delta_{\lambda} \varphi \\
=\frac{1}{2} \sum_{\lambda, \mu \in \Lambda_{1}}\left(\bar{\delta}_{\lambda} \varphi\right)\left(\bar{\delta}_{\lambda} q_{\mu}\right) \Delta_{\mu} \varphi+\sum_{\lambda, \mu \in \Lambda_{1}}\left(\bar{\delta}_{\lambda} \varphi\right)\left(\bar{\delta}_{\lambda} p_{\mu}\right) \delta_{\mu} \varphi \\
+\sum_{\lambda, \mu \in \Lambda_{1}}\left(\bar{\delta}_{\lambda} \varphi\right)\left(\bar{\delta}_{\lambda} \chi_{\mu}\right) \delta_{\mu} \delta_{\lambda} \varphi=: I_{1}^{(1)}+I_{1}^{(2)}+I_{1}^{(3)}, \\
I_{2}=I_{2}^{(1)}+I_{2}^{(2)},
\end{gathered}
$$

where in the notation $\xi=D \varphi /|D \varphi|$ and $\psi_{(\xi)}=\xi_{i} D_{i} \psi$,

$$
\begin{array}{r}
I_{2}^{(1)}=\frac{1}{2} \tau_{0}^{2} \sum_{j=1}^{d} \sum_{\mu \in \Lambda_{1}}\left(D_{j} \varphi\right)\left(D_{j} q_{\mu}\right) \Delta_{\mu} \varphi=\frac{1}{2} \tau_{0}^{2}|D \varphi| \sum_{\mu \in \Lambda_{1}} q_{\mu(\xi)} \Delta_{\mu} \varphi \\
I_{2}^{(2)}=\tau_{0}^{2} \sum_{j=1}^{d} \sum_{\mu \in \Lambda_{1}}\left(D_{j} \varphi\right)\left(D_{j} p_{\mu}\right) \delta_{\mu} \varphi=\tau_{0}^{2}|D \varphi| \sum_{\mu \in \Lambda_{1}} p_{\mu(\xi)} \delta_{\mu} \varphi .
\end{array}
$$

Set

$$
\bar{\tau}:=\max _{\lambda \in \Lambda_{1}} \tau_{\lambda}, \quad \underline{\tau}:=\min _{\lambda \in \Lambda_{1}} \tau_{\lambda}
$$


and observe that $\chi_{\lambda} \geq \kappa / 2>0$ for sufficiently small $h$, and that $c \geq c_{0}>0$. Then by Young's inequality, we obtain

$$
\begin{gathered}
2 m I_{1}^{(j)} \leq \frac{1-\delta}{3} \sum_{\lambda \in \Lambda_{1}} \mathcal{Q}\left(\bar{\delta}_{\lambda} \varphi\right)+N \mathcal{Q}(\varphi) \quad \text { for } j=1,3, \quad 2 m I_{1}^{(2)} \leq N \mathcal{Q}(\varphi) \\
2 m I_{2}^{(1)} \leq \frac{1-\delta}{3} \sum_{\mu \in \Lambda_{1}} \mathcal{Q}\left(\bar{\delta}_{\mu} \varphi\right)+\tau_{0}^{2} N c_{0}^{-1} c \sum_{\lambda \in \Lambda_{2}}\left|\bar{\delta}_{\lambda} \varphi\right|^{2} \\
2 m I_{2}^{(2)} \leq \tau_{0} N c_{0}^{-1} c \sum_{\lambda \in \Lambda}\left|\bar{\delta}_{\lambda} \varphi\right|^{2}
\end{gathered}
$$

where $N$ is a constant depending only on $m, \kappa, \delta, \bar{\tau}, \underline{\tau}$, the number of elements in $\Lambda_{1}$, and on the supremum norm of the gradients of $p_{\lambda}$ and $q_{\lambda}$ in $x$. Summing up these inequalities and taking $\tau_{0}>0$ sufficiently small we get $(2.5)$ with $K_{1}=3 N$, unit operator $\mathcal{K}$, and with $\delta$ as close to 1 as we wish.

This result is obviously applicable to $\bar{\delta}_{\nu} \varphi$ in place of $\varphi$ for any $\nu \in \Lambda$. It follows that for any $\delta \in(0,1)$ and appropriate constant $K_{1}$ we have

$$
\begin{gathered}
m \sum_{\nu \in \Lambda} A_{1}\left(\bar{\delta}_{\nu} \varphi\right) \leq(1-\delta) \sum_{\lambda \in \Lambda^{2}} \mathcal{Q}\left(\bar{\delta}_{\lambda} \varphi\right) \\
+K_{1} \sum_{\lambda \in \Lambda} \mathcal{Q}\left(\bar{\delta}_{\lambda} \varphi\right)+2(1-\delta) c \sum_{\lambda \in \Lambda^{2}}\left|\bar{\delta}_{\lambda} \varphi\right|^{2} .
\end{gathered}
$$

Now we show that Assumption 2.5 holds. Clearly,

$$
\begin{gathered}
\sum_{\lambda \in \Lambda^{2}}\left(\bar{\delta}_{\lambda} \varphi\right) Q_{\lambda} T_{\lambda} \varphi=\sum_{\lambda \in \Lambda_{1}^{2}}\left(\bar{\delta}_{\lambda} \varphi\right) Q_{\lambda} T_{\lambda} \varphi+2 \sum_{\lambda \in \Lambda_{1} \times \Lambda_{2}}\left(\bar{\delta}_{\lambda} \varphi\right) Q_{\lambda} T_{\lambda} \varphi \\
+\sum_{\lambda \in \Lambda_{2}^{2}}\left(\bar{\delta}_{\lambda} \varphi\right) Q_{\lambda} \varphi=I_{1}+I_{2}+I_{3} .
\end{gathered}
$$

Using

$$
\Delta_{\mu} T_{\lambda}=\Delta_{\mu}+h \delta_{\lambda^{1}} \delta_{\lambda^{2}}\left(\delta_{\mu}+\delta_{-\mu}\right)+\left(\delta_{\lambda^{1}}+\delta_{\lambda^{2}}\right)\left(\delta_{\mu}+\delta_{-\mu}\right)
$$

for $\lambda=\left(\lambda^{1}, \lambda^{2}\right) \in \Lambda_{1}^{2}$ and $\mu \in \Lambda_{1}$, we have

$$
I_{1}=\sum_{\lambda \in \Lambda_{1}^{2}}\left(\bar{\delta}_{\lambda} \varphi\right) Q_{\lambda} T_{\lambda} \varphi=I_{1}^{(1)}+I_{1}^{(2)}
$$

with

$$
\begin{gathered}
I_{1}^{(1)}=\frac{1}{2} \sum_{\lambda \in \Lambda_{1}^{2}, \mu \in \Lambda_{1}}\left(\bar{\delta}_{\lambda} \varphi\right)\left(\bar{\delta}_{\lambda} q_{\mu}\right)\left(4 \delta_{\lambda^{1}}-\delta_{-\mu}\right) \delta_{\mu} \varphi \\
I_{1}^{(2)}=h \sum_{\lambda \in \Lambda_{1}^{2}, \mu \in \Lambda_{1}}\left(\bar{\delta}_{\lambda} \varphi\right)\left(\bar{\delta}_{\lambda} q_{\mu}\right) \delta_{\lambda} \delta_{\mu} \varphi
\end{gathered}
$$


As above, we have

$$
\begin{gathered}
\sum_{\lambda \in \Lambda_{1}^{2}}\left|\bar{\delta}_{\lambda} \varphi\right|^{2} \leq N \sum_{\lambda, \mu \in \Lambda_{1}}\left|\delta_{\lambda} \delta_{\mu} \varphi\right|^{2} \leq N \sum_{\lambda \in \Lambda} \mathcal{Q}\left(\bar{\delta}_{\lambda} \varphi\right), \\
\sum_{\lambda \in \Lambda_{1}^{2}, \mu \in \Lambda_{1}}\left|\delta_{\lambda} \delta_{\mu} \varphi\right|^{2} \leq N \sum_{\lambda \in \Lambda^{2}} \mathcal{Q}\left(\bar{\delta}_{\lambda} \varphi\right),
\end{gathered}
$$

and hence, by Young's inequality

$$
\begin{gathered}
n(n-1) I_{1}^{(1)} \leq N \sum_{\lambda \in \Lambda} \mathcal{Q}\left(\bar{\delta}_{\lambda} \varphi\right), \\
n(n-1) I_{1}^{(2)} \leq h^{2} N \sum_{\lambda \in \Lambda^{2}} \mathcal{Q}\left(\bar{\delta}_{\lambda} \varphi\right)+N \sum_{\lambda \in \Lambda} \mathcal{Q}\left(\bar{\delta}_{\lambda} \varphi\right),
\end{gathered}
$$

where $N$ is a constant depending only on $m, \kappa, \bar{\tau}$, the number of elements in $\Lambda_{1}$ and on the supremum norm of $\left|D^{2} q_{\lambda}\right|$. Similarly,

$$
\begin{gathered}
n(n-1) I_{2}=n(n-1) \tau_{0} \sum_{i=1}^{d} \sum_{\nu, \mu \in \Lambda_{1}}\left(\bar{\delta}_{\nu} \tau_{0} D_{i} \varphi\right)\left(\bar{\delta}_{\nu} D_{i} q_{\mu}\right) T_{\nu} \Delta_{\mu} \varphi \\
\leq \tau_{0} N \sum_{\lambda \in \Lambda} \mathcal{Q}(\bar{\delta} \varphi)+N \tau_{0} c \mathcal{K}\left(\sum_{\mu \in \Lambda^{2}} \bar{\delta}_{\mu} \varphi\right), \\
n(n-1) I_{3}=\frac{1}{2} n(n-1) \tau_{0}^{2} \sum_{i, j=1}^{d} \sum_{\mu \in \Lambda_{1}}\left(\tau_{0}^{2} D_{i j} \varphi\right)\left(D_{i j} q_{\mu}\right) \Delta_{\mu} \varphi \\
\leq N \tau_{0}^{2} c \sum_{\lambda \in \Lambda^{2}}\left|\bar{\delta}_{\lambda} \varphi\right|^{2},
\end{gathered}
$$

where $N$ denote some constants depending on $m, d, c_{0}, \kappa, \bar{\tau}, \underline{\tau}$, the number of elements in $\Lambda_{1}$ and on the supremum norm of $\left|D^{2} q_{\lambda}\right|$. Summing up the inequalities (2.17) through (2.21) and choosing $\tau_{0}$ and $h_{0}$ sufficiently small we obtain (2.6).

3. Proof of Theorem 2.1. For $m=1$ estimate (2.7) holds by virtue of Theorem 2.1 from [14], proved by the aid of the following version of the maximum principle (Corollary 3.2 in [14]).

Lemma 3.1. Let Assumption 2.1 with $m=0$ be satisfied and let $\chi_{\lambda} \geq 0$ for all $\lambda \in \Lambda_{1}$. Let $v$ be a bounded function on $H_{T}$, such that the partial derivative $D_{t} v:=\partial v(t, x) / \partial t$ exists in $H_{T}$. Let $F$ be a nonnegative integrable function on $[0, T]$, and let $C$ be a nonnegative bounded function on $H_{T}$ such that

$$
\nu:=\sup _{H_{T}}(C-c)<0
$$


Assume that for all $(t, x) \in H_{T}$ we have

$$
D_{t} v \leq L v+C \bar{v}_{+}+F,
$$

where $\bar{v}(t)=\sup \left\{v(t, x): x \in \mathbb{R}^{d}\right\}$. Then in $[0, T]$ we have

$$
\bar{v}(t) \leq \bar{v}_{+}(0)+|\nu|^{-1} \sup _{[0, t]} F
$$

where $a_{+}:=(|a|+a) / 2$ for real numbers $a$.

For the proof of this lemma we refer to [14]. In order to obtain Theorem 2.1 for $m \geq 2$ we need some more lemmas. First we prove a lemma which will be used a few times in the future. By $\mathcal{K}$ in the lemma and later in the article we mean a generic operator of class $\mathfrak{K}$. This operator may change each time it is mentioned even in one line (cf. the use of $o(n)$ ). Thus, for example, for nonnegative functions $\alpha, \beta$ on $\mathbb{R}^{d}$ the formula $\alpha \mathcal{K}+\beta \mathcal{K}=(\alpha+\beta) \mathcal{K}$ means the simple fact that for any $\mathcal{K}_{1}, \mathcal{K}_{2} \in \mathfrak{K}$

$$
\alpha \mathcal{K}_{1}+\beta \mathcal{K}_{2}=(\alpha+\beta) \mathcal{K}_{3}
$$

with

$$
\mathcal{K}_{3}:=\frac{\alpha}{\alpha+\beta} \mathcal{K}_{1}+\frac{\beta}{\alpha+\beta} \mathcal{K}_{2} \in \mathfrak{K} \quad\left(\frac{0}{0}:=0\right) .
$$

Lemma 3.2. Let Assumption 2.1 be satisfied. Let $n \geq 1$ be an integer. Set

$$
A^{2}=\left\|\Lambda_{1}\right\|^{2}+\tau_{0}^{2} .
$$

Then for any $\varphi \in C^{n}$ we have

$$
\sum_{\lambda \in \Lambda_{1}^{n}}\left|\bar{\delta}_{\lambda} \varphi\right|^{2} \leq\left\|\Lambda_{1}\right\|^{2 n} \mathcal{K}\left(\left|D^{n} \varphi\right|^{2}\right), \quad \sum_{\lambda \in \Lambda^{n}}\left|\bar{\delta}_{\lambda} \varphi\right|^{2} \leq A^{2 n} \mathcal{K}\left(\left|D^{n} \varphi\right|^{2}\right) .
$$

Furthermore, if $1 \leq n \leq m$, then for any $\varphi \in C^{n}$

$$
\begin{gathered}
\sum_{\lambda \in \Lambda^{n}}\left|P_{\lambda} T_{\lambda} \varphi\right|^{2} \leq\left|\Lambda_{1}\right|^{2} A^{2 n}\left(\sup _{H_{T}} \sum_{\mu \in \Lambda_{1}}\left|D^{n} p_{\mu}\right|^{2}\right) \mathcal{K}\left(|D \varphi|^{2}\right) \\
\leq \tau_{0}^{-2}\left|\Lambda_{1}\right|^{2} A^{2 n}\left(\sup _{H_{T}} \sum_{\mu \in \Lambda_{1}}\left|D^{n} p_{\mu}\right|^{2}\right) \mathcal{K}\left(\sum_{\lambda \in \Lambda}\left|\bar{\delta}_{\lambda} \varphi\right|^{2}\right),
\end{gathered}
$$

and if assumption (2.3) holds,

$$
\begin{gathered}
\sum_{\lambda \in \Lambda^{n}}\left|Q_{\lambda} T_{\lambda} \varphi\right|^{2} \leq\left|\Lambda_{1}\right|^{4} A^{2 n}\left(\sup _{H_{T}} \sum_{\mu \in \Lambda_{1}}\left|D^{n} q_{\mu}\right|^{2}\right) \mathcal{K}\left(\left|D^{2} \varphi\right|^{2}\right) \\
\leq \tau_{0}^{-4}\left|\Lambda_{1}\right|^{4} A^{2 n}\left(\sup _{H_{T}} \sum_{\mu \in \Lambda_{1}}\left|D^{n} q_{\mu}\right|^{2}\right) \mathcal{K}\left(\sum_{\lambda \in \Lambda^{2}}\left|\bar{\delta}_{\lambda} \varphi\right|^{2}\right) .
\end{gathered}
$$

Finally,

$$
\left(\sum_{\lambda \in \Lambda_{1}} q_{\lambda}\left(\delta_{\lambda} \varphi\right)^{2}\right)^{2} \leq M_{0}^{2} \sum_{\lambda \in \Lambda_{1}}\left(\delta_{\lambda} \varphi\right)^{4} \leq M_{0}^{2}\left(\sum_{\lambda \in \Lambda_{1}}\left(\delta_{\lambda} \varphi\right)^{2}\right)^{2}
$$


Proof. It is easy to see that for $\lambda \in \Lambda_{1}^{n}$ we have

$$
\bar{\delta}_{\lambda} \varphi(x)=h^{-n} \int_{[0, h]^{n}} \varphi_{\lambda}(\theta, x) d \theta
$$

where, for $y(\lambda, \theta)=\lambda^{1} \theta^{1}+\ldots+\lambda^{n} \theta^{n}$ and $\tau_{\lambda}=\tau_{\lambda^{1}} \tau_{\lambda^{2}} \cdot \ldots \cdot \tau_{\lambda^{n}}$,

$$
\varphi_{\lambda}(\theta, x)=\tau_{\lambda} \sum_{i_{1}, \ldots, i_{n}=1}^{d} \lambda_{i_{1}}^{1} \cdot \ldots \cdot \lambda_{i_{n}}^{n} D_{i_{1}} \cdot \ldots \cdot D_{i_{n}} \varphi(x+y(\lambda, \theta)) .
$$

By Cauchy's inequality

$$
\begin{aligned}
& \left|\bar{\delta}_{\lambda} \varphi(x)\right|^{2} \leq h^{-n} \int_{[0, h]^{n}}\left|\varphi_{\lambda}(\theta, x)\right|^{2} d \theta, \\
& \left|\varphi_{\lambda}(\theta, x)\right| \leq \tau_{\lambda}|\lambda|\left|D^{n} \varphi(x+y(\lambda, \theta))\right|,
\end{aligned}
$$

where $|\lambda|:=\left|\lambda^{1}\right| \cdot \ldots \cdot\left|\lambda^{n}\right|$. It follows that the first inequality in (3.3) holds with with $\mathcal{D}_{h}$ in place of $\mathcal{K}$, where

$$
\mathcal{D}_{h} \psi(x)=\left\|\Lambda_{1}\right\|^{-2 n} \sum_{\lambda \in \Lambda_{1}^{n}} \tau_{\lambda}^{2}|\lambda|^{2} h^{-n} \int_{[0, h]^{n}} \psi(x+y(\lambda, \theta)) d \theta \quad\left(\frac{1}{0}:=0\right) .
$$

Since

$$
\sum_{\lambda \in \Lambda_{1}^{n}} \tau_{\lambda}^{2}|\lambda|^{2}=\left\|\Lambda_{1}\right\|^{2 n}
$$

$\mathcal{D}_{h} 1 \leq 1$, that is $\mathcal{D}_{h} \in \mathfrak{K}$ and the first inequality in (3.3) is proved.

To prove the second one introduce $\mathcal{D}_{h, k}$ as the operators for which first inequality in (3.3) holds with $k$ in place of $n$, recall that $\Lambda_{1}^{0}=\Lambda_{2}^{0}=\{0\}$ and $\bar{\delta}_{0}$ is the identity operator, and observe that the left-hand side of the second inequality equals

$$
\begin{aligned}
& \sum_{k=0}^{n} C_{n}^{k} \sum_{\lambda \in \Lambda_{1}^{k}, \mu \in \Lambda_{2}^{n-k}}\left|\bar{\delta}_{\lambda} \bar{\delta}_{\mu} \varphi\right|^{2} \leq \sum_{k=0}^{n} C_{n}^{k}\left\|\Lambda_{1}\right\|^{2 k} \mathcal{D}_{h, k}\left(\sum_{\mu \in \Lambda_{2}^{n-k}}\left|\bar{\delta}_{\mu} D^{k} \varphi\right|^{2}\right) \\
& =\sum_{k=0}^{n} C_{n}^{k}\left\|\Lambda_{1}\right\|^{2 k} \tau_{0}^{2(n-k)} \mathcal{D}_{h, k}\left(\left|D^{n} \varphi\right|^{2}\right)=:\left(\left\|\Lambda_{1}\right\|^{2}+\tau_{0}^{2}\right)^{n} \mathcal{E}_{h}\left(\left|D^{n} \varphi\right|^{2}\right),
\end{aligned}
$$

with $\mathcal{E}_{h} \in \mathfrak{K}$. This proves the first assertion of the lemma.

To prove (3.4) notice that by Cauchy's inequality and (3.3) for $\lambda \in \Lambda^{n}$

$$
\begin{gathered}
\left|P_{\lambda} T_{\lambda} \varphi\right|^{2}=\left|\sum_{\mu \in \Lambda_{1}}\left(\bar{\delta}_{\lambda} p_{\mu}\right) T_{\lambda} \delta_{\mu} \varphi\right|^{2} \\
\leq \sum_{\mu \in \Lambda_{1}}\left(\bar{\delta}_{\lambda} p_{\mu}\right)^{2} T_{\lambda} \sum_{\mu \in \Lambda_{1}}\left(\delta_{\mu} \varphi\right)^{2} \leq\left|\Lambda_{1}\right|^{2} \sum_{\mu \in \Lambda_{1}}\left(\bar{\delta}_{\lambda} p_{\mu}\right)^{2} T_{\lambda} \mathcal{D}_{h, 1}\left(|D \varphi|^{2}\right) .
\end{gathered}
$$


Hence the left-hand side of (3.4) is less than

$$
\begin{gathered}
\left|\Lambda_{1}\right|^{2} \sum_{\mu \in \Lambda_{1}, \lambda \in \Lambda^{n}}\left(\bar{\delta}_{\lambda} p_{\mu}\right)^{2} T_{\lambda} \mathcal{D}_{h, 1}\left(|D \varphi|^{2}\right) \\
=:\left|\Lambda_{1}\right|^{2} A^{2 n}\left(\sup _{H_{T}} \sum_{\mu \in \Lambda_{1}}\left|D^{n} p_{\mu}\right|^{2}\right) \mathcal{H}_{h}\left(|D \varphi|^{2}\right) .
\end{gathered}
$$

Here $\mathcal{H}_{h} \in \mathfrak{K}$, since by (3.3)

$$
\sum_{\mu \in \Lambda_{1}, \lambda \in \Lambda^{n}}\left(\bar{\delta}_{\lambda} p_{\mu}\right)^{2} \leq A^{2 n} \mathcal{D}_{h, n}\left(\sum_{\mu \in \Lambda_{1}}\left|D^{n} p_{\mu}\right|^{2}\right) \leq A^{2 n} \sup _{H_{T}} \sum_{\mu \in \Lambda_{1}}\left|D^{n} p_{\mu}\right|^{2} .
$$

This proves (3.4). To prove (3.5), notice that $\sum_{\mu \in \Lambda_{1}} \mu \delta_{\lambda} q_{\mu}=0$, which implies that

$$
Q_{\lambda} T_{\lambda} \varphi=h^{-1} \sum_{\mu \in \Lambda_{1}}\left(\bar{\delta}_{\lambda} q_{\mu}\right) T_{\lambda}\left(\delta_{\mu} \varphi-\mu_{i} D_{i} \varphi\right)=\sum_{\mu \in \Lambda_{1}}\left(\bar{\delta}_{\lambda} q_{\mu}\right) T_{\lambda} \psi_{\mu}
$$

where

$$
\psi_{\mu}=h^{-1}\left(\delta_{\mu} \varphi-\mu_{i} D_{i} \varphi\right)
$$

Hence as above the left-hand side of (3.5) is less than

$$
\sum_{\lambda \in \Lambda^{n}} \sum_{\mu \in \Lambda_{1}}\left(\bar{\delta}_{\lambda} q_{\mu}\right)^{2} T_{\lambda} \sum_{\mu \in \Lambda_{1}}\left|\psi_{\mu}\right|^{2}=: A^{2 n}\left(\sup _{H_{T}} \sum_{\mu \in \Lambda_{1}}\left|D^{n} q_{\mu}\right|^{2}\right) \mathcal{F}_{h}\left(\sum_{\mu \in \Lambda_{1}}\left|\psi_{\mu}\right|^{2}\right),
$$

where $\mathcal{F}_{h} \in \mathfrak{K}$. Furthermore,

$$
\begin{gathered}
\psi_{\mu}(x)=h^{-2} \int_{0}^{h}(h-\theta) \mu_{i} \mu_{j} D_{i j} \varphi(x+\mu \theta) d \theta \\
\leq|\mu|^{2} h^{-1} \int_{0}^{h}\left|D^{2} \varphi(x+\mu \theta)\right| d \theta=|\mu|^{2} \int_{0}^{1}\left|D^{2} \varphi(x+h \mu \theta)\right| d \theta, \\
\left|\psi_{\mu}(x)\right|^{2} \leq|\mu|^{4} \int_{0}^{1}\left|D^{2} \varphi(x+h \mu \theta)\right|^{2} d \theta,
\end{gathered}
$$

and we obtain (3.5) with the operator

$$
\mathcal{K} \psi:=\left|\Lambda_{1}\right|^{-4} \mathcal{F}_{h}\left(\sum_{\mu \in \Lambda_{1}}|\mu|^{4} \int_{0}^{1} \psi(\cdot+h \mu \theta) d \theta\right),
$$

which is in $\mathfrak{K}$ because

$$
\mathcal{K} 1 \leq\left|\Lambda_{1}\right|^{-4} \sum_{\mu \in \Lambda_{1}}|\mu|^{4} \leq 1 .
$$

Since the last assertion of the lemma is obvious, the lemma is proved. 
The following lemma can be proved easily by induction on $n$. (Sums over empty sets of indices are defined to be 0 in the lemma, and everywhere in the article.)

Lemma 3.3. Let $n \geq 1$ be an integer, $\psi$ and $\varphi$ be $n$ times continuously differentiable functions on $\mathbb{R}^{d}$, and $\lambda \in \Lambda^{n}$. Then

$$
\begin{gathered}
\bar{\delta}_{\lambda}(\psi \varphi)=\psi \bar{\delta}_{\lambda} \varphi+\sum_{i=1}^{n}\left(\bar{\delta}_{\lambda^{i}} \psi\right) \bar{\delta}_{\bar{\lambda}(i)} T_{\lambda^{i}} \varphi \\
+\sum_{1 \leq i<j \leq n}\left(\bar{\delta}_{\lambda(i, j)} \psi\right) \bar{\delta}_{\bar{\lambda}(i, j)} T_{\lambda(i, j)} \varphi+\ldots \\
+\sum_{1 \leq i_{1}<\ldots<i_{k} \leq n}\left(\bar{\delta}_{\lambda\left(i_{1}, \ldots, i_{k}\right)} \psi\right) \bar{\delta}_{\bar{\lambda}\left(i_{1}, \ldots, i_{k}\right)} T_{\lambda\left(i_{1}, \ldots, i_{k}\right)} \varphi \\
+\ldots+\left(\bar{\delta}_{\lambda} \psi\right) T_{\lambda} \varphi,
\end{gathered}
$$

where $\lambda\left(i_{1}, \ldots, i_{k}\right)=\left(\lambda^{i_{1}}, \ldots, \lambda^{i_{k}}\right), \bar{\lambda}\left(i_{1}, \ldots, i_{k}\right)$ is the sequence of vectors $\lambda^{1}, \ldots, \lambda^{n}$ from which the vectors standing on the places with numbers $i_{1}, \ldots, i_{k}$ are removed, and $\bar{\delta}_{\bar{\lambda}(1)}:=1$ for $n=1$.

Proof of Theorem 2.1. Recall that $\Lambda^{0}=\{0\}$ and $\bar{\delta}_{0}=T_{0}$ is the unit operator. Fix $h \in\left(0, h_{0}\right]$, for $0 \leq k \leq m$ set

$$
u=u_{h}, \quad f=f_{h}, \quad V_{0}=u^{2}, \quad V_{k}=\sum_{\lambda \in \Lambda^{k}}\left|\bar{\delta}_{\lambda} u\right|^{2}, \quad \bar{V}_{k}(t)=\sup _{\mathbb{R}^{d}} V_{k}(t, x),
$$

and recall that $F_{n}$ is introduced in Theorem 2.1. Take an integer $n \in[1, m]$. Then we have

$$
L_{h}^{0} V_{n}=2 \sum_{\lambda \in \Lambda^{n}}\left(\bar{\delta}_{\lambda} u\right) L_{h}^{0} \bar{\delta}_{\lambda} u+\sum_{\lambda \in \Lambda^{n}} \mathcal{Q}\left(\bar{\delta}_{\lambda} u\right)
$$

By Lemma 3.3

$$
2 \sum_{\lambda \in \Lambda^{n}}\left(\bar{\delta}_{\lambda} u\right) L_{h}^{0} \bar{\delta}_{\lambda} u=2 \sum_{\lambda \in \Lambda^{n}}\left(\bar{\delta}_{\lambda} u\right) \bar{\delta}_{\lambda} L_{h}^{0} u-\sum_{n \geq k \geq 1} I_{n, k},
$$

where

$$
I_{n, k}:=C_{n}^{k} \sum_{\mu \in \Lambda^{n-k}} A_{k}\left(\bar{\delta}_{\mu} u\right)
$$

By Assumption 2.4,

$$
\begin{aligned}
n A_{1}\left(\bar{\delta}_{\mu} u\right) \leq(1-\delta) \sum_{\lambda \in \Lambda} \mathcal{Q}\left(\bar{\delta}_{\lambda} \bar{\delta}_{\mu} u\right)+K_{1} \mathcal{Q}\left(\bar{\delta}_{\mu} u\right) \\
+2(1-\delta) c \mathcal{K}\left(\sum_{\lambda \in \Lambda}\left|\bar{\delta}_{\lambda} \bar{\delta}_{\mu} u\right|^{2}\right) .
\end{aligned}
$$


Hence,

$$
I_{n, 1} \leq(1-\delta) \sum_{\lambda \in \Lambda^{n}} \mathcal{Q}\left(\bar{\delta}_{\lambda} u\right)+K_{1} \sum_{\lambda \in \Lambda^{n-1}} \mathcal{Q}\left(\bar{\delta}_{\lambda} u\right)+2(1-\delta) c \bar{V}_{n}
$$

Next, if $n \geq 2$, then

$$
\begin{aligned}
I_{n, 1} & +I_{n, 2}=n \sum_{\mu \in \Lambda^{n-1}} A_{1}\left(\bar{\delta}_{\mu} u\right)+\frac{1}{2} n(n-1) \sum_{\mu \in \Lambda^{n-2}} A_{2}\left(\bar{\delta}_{\mu} u\right) \\
& =\sum_{\mu \in \Lambda^{n-2}}\left(n \sum_{\nu \in \Lambda} A_{1}\left(\bar{\delta}_{\nu}\left(\bar{\delta}_{\mu} u\right)\right)+\frac{1}{2} n(n-1) A_{2}\left(\bar{\delta}_{\mu} u\right)\right)
\end{aligned}
$$

so that by Assumption 2.5

$$
\begin{gathered}
I_{n, 1}+I_{n, 2} \leq n(n-1) \sum_{\mu \in \Lambda^{n-2}} \sum_{\lambda \in \Lambda^{2}}\left(\bar{\delta}_{\lambda} \bar{\delta}_{\mu} u\right) P_{\lambda} T_{\lambda} \bar{\delta}_{\mu} u \\
+(1-\delta) \sum_{\lambda \in \Lambda^{n}} \mathcal{Q}\left(\bar{\delta}_{\lambda} u\right)+K_{1} \sum_{\lambda \in \Lambda^{n-1}} \mathcal{Q}\left(\bar{\delta}_{\lambda} u\right)+2(1-\delta) c \bar{V}_{n}+K_{1} \bar{V}_{n-1} .
\end{gathered}
$$

By Lemma 3.2

$$
n(n-1) \sum_{\mu \in \Lambda^{n-2}} \sum_{\lambda \in \Lambda^{2}}\left(\bar{\delta}_{\lambda} \bar{\delta}_{\mu} u\right) P_{\lambda} T_{\lambda} \bar{\delta}_{\mu} u+\sum_{n \geq k \geq 3} I_{n, k} \leq \delta^{2} V_{n}+N \sum_{k=1}^{n-1} \bar{V}_{k},
$$

where and below by the sum over an empty set we mean zero. It follows that, for $n \in[1, m]$,

$$
\begin{gathered}
L_{h}^{0} V_{n} \geq 2 \sum_{\lambda \in \Lambda^{n}}\left(\bar{\delta}_{\lambda} u\right) \bar{\delta}_{\lambda} L_{h}^{0} u+\delta \sum_{\lambda \in \Lambda^{n}} \mathcal{Q}\left(\bar{\delta}_{\lambda} u\right)-K_{1} \sum_{\lambda \in \Lambda^{n-1}} \mathcal{Q}\left(\bar{\delta}_{\lambda} u\right) \\
-\left(2 c-2 \delta c+\delta^{2}\right) \bar{V}_{n}-N \sum_{k=1}^{n-1} \bar{V}_{k}
\end{gathered}
$$

Next,

$$
2 \sum_{\lambda \in \Lambda^{n}}\left(\bar{\delta}_{\lambda} u\right) \bar{\delta}_{\lambda} L_{h}^{0} u=D_{t} V_{n}+R_{1}-R_{2}
$$

where

$$
R_{1}:=2 \sum_{\lambda \in \Lambda^{n}}\left(\bar{\delta}_{\lambda} u\right) \bar{\delta}_{\lambda}(c u), \quad R_{2}:=2 \sum_{\lambda \in \Lambda^{n}}\left(\bar{\delta}_{\lambda} u\right) \bar{\delta}_{\lambda} f .
$$

Similarly to $(3.8)$

$$
R_{1}=2 c V_{n}+2 \sum_{k=1}^{n} C_{n}^{k} R_{1 k}
$$


where

$$
R_{1 k}:=\sum_{\lambda \in \Lambda^{n-k}} \sum_{\mu \in \Lambda^{k}}\left(\bar{\delta}_{\mu} \bar{\delta}_{\lambda} u\right)\left(\bar{\delta}_{\mu} c\right) T_{\mu}\left(\bar{\delta}_{\lambda} u\right) .
$$

By our assumptions, (3.3), and Cauchy's inequality

$$
\begin{gathered}
\sum_{\lambda \in \Lambda^{n-k}}\left|\bar{\delta}_{\mu} \bar{\delta}_{\lambda} u\right| T_{\mu}\left|\bar{\delta}_{\lambda} u\right| \leq\left(\sum_{\lambda \in \Lambda^{n-k}}\left(\bar{\delta}_{\mu} \bar{\delta}_{\lambda} u\right)^{2}\right)^{1 / 2} T_{\mu}\left(\sum_{\lambda \in \Lambda^{n-k}}\left(\bar{\delta}_{\lambda} u\right)^{2}\right)^{1 / 2} \\
\leq \bar{V}_{n-k}^{1 / 2}\left(\sum_{\lambda \in \Lambda^{n-k}}\left(\bar{\delta}_{\mu} \bar{\delta}_{\lambda} u\right)^{2}\right)^{1 / 2} \\
\left|R_{1 k}\right| \leq \bar{V}_{n-k}^{1 / 2} V_{n}^{1 / 2}\left(\sum_{\mu \in \Lambda^{k}}\left|\bar{\delta}_{\mu} c\right|^{2}\right)^{1 / 2} \leq A_{H_{T}^{k}}\left(\sup _{H^{\prime}}\left|D^{k} c\right|\right) V_{n}^{1 / 2} \bar{V}_{n-k}^{1 / 2}
\end{gathered}
$$

Cauchy's inequality also allows us to estimate $R_{2}$ and conclude from (3.10) that, for $n \in[1, m]$,

$$
\begin{gathered}
L_{h}^{0} V_{n}-2 c V_{n}-D_{t} V_{n} \geq \delta \mathcal{Q}_{n} \\
-K_{1} \mathcal{Q}_{n-1}-\left(2 c-2 \delta c+2 \delta^{2}\right) \bar{V}_{n}-N \sum_{k=0}^{n-1} \bar{V}_{k}-N F_{n}^{2},
\end{gathered}
$$

where

$$
\mathcal{Q}_{k}=\sum_{\lambda \in \Lambda^{k}} \mathcal{Q}\left(\bar{\delta}_{\lambda} u\right)
$$

We now prove (2.7) by showing that for each $n \in[1, m]$

$$
V_{k} \leq N\left(F_{k}^{2}+G_{k}^{2}\right), \quad k=0,1, \ldots, n .
$$

We prove this by induction on $n$. By Lemma 3.1 we have

$$
V_{0} \leq N\left(F_{0}^{2}+G_{0}^{2}\right)
$$

Using this, from (3.12) we obtain (see more details in [14])

$$
V_{1} \leq N\left(F_{1}^{2}+G_{1}^{2}\right)
$$

by Lemma 3.1, provided that $0 \leq 2 c-2 \delta c+2 \delta^{2} \leq 2 c-\delta^{2}$ which is true indeed if

$$
3 \delta \leq 2 c_{0}
$$

This may look like a nontrivial restriction on $\delta$. However, obviously, if our assumptions are satisfied with a $\delta \in(0,1)$, they are also satisfied with any $\delta^{\prime} \in(0, \delta]$. Therefore, without losing generality we suppose that (3.14) is valid. Thus we have obtained (3.13) for $n=1$. Let $n \geq 2$ and assume that (3.13) holds with $n-1$ in place of $n$. Then from (3.12) for $k=1, \ldots, n$ we get

$$
L_{h}^{0} V_{k}-2 c V_{k}-D_{t} V_{k} \geq \delta \mathcal{Q}_{k}-K_{1} \mathcal{Q}_{k-1}-C_{\delta} I_{k=n} \bar{V}_{n}-N\left(F_{n}^{2}+G_{n}^{2}\right),
$$


with $C_{\delta}=2\left(c-\delta c+\delta^{2}\right)$. Actually, (3.15) is true also for $k=0$ if we set $\mathcal{Q}_{-1}=0$, since

$$
\begin{gathered}
L_{h}^{0}\left(u^{2}\right)-2 c u^{2}-D_{t}\left(u^{2}\right)=2 u\left(L_{h}^{0} u-c u-D_{t} u\right)+\mathcal{Q}_{0}(u) \\
=-2 u f+\mathcal{Q}_{0}(u) \geq-N\left(F_{1}^{2}+G_{1}^{2}\right)+\mathcal{Q}_{0}(u) .
\end{gathered}
$$

Next we set $\mu=K_{1} / \delta$, multiply (3.15) by $\mu^{n-k}$ and sum up the resulting inequalities with respect to $k=0, \ldots, n$. Then, for

$$
W_{n}:=\sum_{k=0}^{n} \mu^{n-k} V_{k},
$$

we obtain

$$
\begin{gathered}
L_{h}^{0} W_{n}-2 c W_{n}-D_{t} W_{n} \geq \delta \mathcal{Q}_{n}-C_{\delta} \bar{V}_{n}-N\left(F_{n}^{2}+G_{n}^{2}\right) \\
\geq-C_{\delta} \bar{W}_{n}-N\left(F_{n}^{2}+G_{n}^{2}\right) .
\end{gathered}
$$

Recalling (3.14) and using Lemma 3.1 shows that (3.13) holds. This justifies the induction and proves the theorem.

\section{Discussion of Assumptions 2.4 and 2.5.}

REMARK 4.1. It may be instructive to see what happens with Assumption 2.4 as $h \downarrow 0$. We suppose that Assumption 2.1 and condition (S) are satisfied and $m=1$. For simplicity we concentrate on the case that $\tau_{0}=\tau_{\lambda} \equiv 1$.

Take a smooth function $\varphi$ and at a fixed point of $H_{T}$ let $h \downarrow 0$ in (2.5). Since all terms apart, possibly, from $\mathcal{K}$ involve the values of $\varphi$ and its derivatives only at the chosen point the last term in $(2.5)$ will become $2(1-\delta) c\left(A^{-1} \xi, \xi\right)$, where

$$
A=\sum_{\lambda \in \Lambda} \lambda \lambda^{*}, \quad \xi=A D \varphi
$$

$\lambda^{*}$ is a row vector transpose of $\lambda$ and $D \varphi$ is a column vector of the first-order partial derivatives. Also introduce

$$
a=\sum_{\lambda \in \Lambda_{1}} \lambda \lambda^{*} q_{\lambda}, \quad b=\sum_{\lambda \in \Lambda_{1}} \lambda p_{\lambda} .
$$

Then as is easy to see for $h \downarrow 0$ we have

$$
\begin{aligned}
& A_{1}(\varphi) \rightarrow \sum_{\lambda \in \Lambda}(D \varphi)^{*} \lambda \sum_{\mu \in \Lambda_{1}}\left[\lambda^{*} D q_{\mu} \mu^{*} D^{2} \varphi \mu+2 \lambda^{*} D p_{\mu} \mu^{*} D \varphi\right] \\
= & \xi^{i}\left[\operatorname{tr}\left(\left(D_{i} a\right) D^{2} \varphi\right)+2\left(b, A^{-1} \xi\right)\right]=\operatorname{tr}\left(a_{(\xi)} D^{2} \varphi\right)+2\left(b_{(\xi)}, A^{-1} \xi\right),
\end{aligned}
$$

where $D^{2} \phi$ is the Hessian matrix of $\varphi$ and we use the notation $u_{(\xi)}=(\xi, D u)$. Next,

$$
\begin{aligned}
& \mathcal{Q}(\varphi) \rightarrow\left(\xi, A^{-1} a A^{-1} \xi\right), \\
& \sum_{\lambda \in \Lambda} \mathcal{Q}\left(\delta_{\lambda} \varphi\right) \rightarrow \sum_{\lambda \in \Lambda} \sum_{\mu \in \Lambda_{1}} q_{\mu} \lambda^{*} D^{2} \varphi \mu \mu^{*} D^{2} \varphi \lambda=\operatorname{tr} A\left(D^{2} \varphi\right) a D^{2} \varphi
\end{aligned}
$$


and (2.5) for $m=1$ becomes

$$
\begin{gathered}
\operatorname{tr}\left(a_{(\xi)} D^{2} \varphi\right)+2\left(b_{(\xi)}, A^{-1} \xi\right) \leq(1-\delta) \operatorname{tr} A\left(D^{2} \varphi\right) a D^{2} \varphi \\
+K_{1}\left(\xi, A^{-1} a A^{-1} \xi\right)+2(1-\delta) c\left(A^{-1} \xi, \xi\right) .
\end{gathered}
$$

At our fixed point the values of $D^{2} \varphi$ have no relation to $\xi$. This implies that $\operatorname{tr} A B a B \geq 0$ for any symmetric matrix $B$. For $B=\eta \eta^{*}$ with nonzero $\eta \in \mathbb{R}^{d}$ this yields $\operatorname{tr} A \eta \eta^{*} a \eta \eta^{*}=\operatorname{tr} \eta^{*} A \eta \eta^{*} a \eta=(A \eta, \eta)(a \eta, \eta) \geq 0$ and since $(A \eta, \eta)>0$, the matrix $a$ is nonnegative. Furthermore, simple manipulations show that

$$
\begin{gathered}
\sup _{B}\left[\operatorname{tr} a_{(\xi)} B-(1-\delta) \operatorname{tr} A B a B\right] \\
=\frac{1}{4(1-\delta)} \lim _{\varepsilon \downarrow 0} \operatorname{tr} A^{-1} a_{(\xi)}(a+\varepsilon I)^{-1} a_{(\xi)}=: \frac{1}{4(1-\delta)} \operatorname{tr} A^{-1} a_{(\xi)} a^{-1} a_{(\xi)},
\end{gathered}
$$

where $I$ is the unit matrix and $a^{-1}$ is the pseudo inverse of $a$. Thus we come to the condition

$$
\begin{aligned}
& \frac{1}{4(1-\delta)} \operatorname{tr} A^{-1} a_{(\xi)} a^{-1} a_{(\xi)}+2\left(b_{(\xi)}, A^{-1} \xi\right) \\
& \leq K_{1}\left(\xi, A^{-1} a A^{-1} \xi\right)+2(1-\delta) c\left(A^{-1} \xi, \xi\right),
\end{aligned}
$$

which should hold for all vectors $\xi \in \mathbb{R}^{d}$. Condition (4.2) is much easier to analyze than (2.5) but unfortunately (4.2) alone is not enough to obtain our estimates for finite-difference equations.

In [14] there are many sufficient conditions for Assumption 2.4 to be satisfied. In the rest of this section we suppose that only Assumptions 2.1 and 2.3 are satisfied and $m \geq 2$. Assume also that for a number $\bar{\tau}>0$ we have that, for any $\lambda \in \Lambda_{1}$,

$$
\text { either } \quad \tau_{\lambda} \geq \bar{\tau} \quad \text { or } \quad D q_{\lambda}(t, x)=D p_{\lambda}(t, x)=0 \text { for all }(t, x) .
$$

In other words this condition says that if $\tau_{\lambda}=0$ for a $\lambda \in \Lambda_{1}$ then for that $\lambda$ the functions $q_{\lambda}$ and $p_{\lambda}$ do not depend on $x \in \mathbb{R}^{d}$.

Recall that by $\mathcal{K}$ we denote a generic operator from class $\mathfrak{K}$, which may depend on $h$ and $t$, and may change each time it is mentioned even in one line.

REMARK 4.2. Assume that $m \geq 2, \Lambda_{1}=-\Lambda_{1}, q_{\lambda}=q_{-\lambda}$ and that for a constant $\theta>0$ we have $q_{\lambda} \leq \theta \chi_{\lambda}\left(=\theta\left(q_{\lambda}+h p_{\lambda}\right)\right)$ for all $h \in\left(0, h_{0}\right]$ and $\lambda \in \Lambda_{1}$. Then, since $q_{\lambda}$ are twice continuously differentiable in $x$ and nonnegative by Assumption 2.3 , we know that $r_{\lambda}:=\sqrt{q_{\lambda}}$ is Lipschitz continuous in $x$ with the Lipschitz constant independent of $t$.

In this situation the following may be useful. Conditions (2.5) and (2.6) involve a mixture of finite differences and derivatives. Therefore, it is reasonable to try to find conditions in terms only of finite differences which would imply (2.5) and (2.6).

We claim that (2.5) and (2.6) are satisfied with a $\tau_{0}>0$ and, perhaps, different $\delta, \mathcal{K}, K_{1}$ if for all smooth $\varphi$ on $H_{T}$ and $n=1, \ldots, m$ we have

$$
2 m \sum_{\lambda \in \Lambda_{1}}\left(\bar{\delta}_{\lambda} \varphi\right) L_{\lambda}^{0} T_{\lambda} \varphi \leq(1-\delta) \sum_{\lambda \in \Lambda_{1}} \mathcal{Q}\left(\bar{\delta}_{\lambda} \varphi\right)
$$




$$
\begin{gathered}
+K_{1} \mathcal{Q}(\varphi)+(1-\delta) c \mathcal{K}\left(\sum_{\lambda \in \Lambda_{1}}\left|\bar{\delta}_{\lambda} \varphi\right|^{2}\right) \\
2 n \sum_{\lambda, \nu \in \Lambda_{1}}\left(\bar{\delta}_{\lambda} \bar{\delta}_{\nu} \varphi\right) L_{\lambda}^{0} T_{\lambda} \bar{\delta}_{\nu} \varphi+n(n-1) \sum_{\lambda \in \Lambda_{1}^{2}}\left(\bar{\delta}_{\lambda} \varphi\right) Q_{\lambda} T_{\lambda} \varphi \\
\leq(1-\delta) \sum_{\nu \in \Lambda_{1}^{2}} \mathcal{Q}\left(\bar{\delta}_{\nu} \varphi\right)+K_{1} \sum_{\nu \in \Lambda_{1}} \mathcal{Q}\left(\bar{\delta}_{\nu} \varphi\right) \\
+(1-\delta) c \mathcal{K}\left(\sum_{\lambda \in \Lambda_{1}^{2}}\left|\bar{\delta}_{\lambda} \varphi\right|^{2}\right)+K_{1} \mathcal{K}\left(\sum_{\lambda \in \Lambda_{1}}\left|\bar{\delta}_{\lambda} \varphi\right|^{2}\right) .
\end{gathered}
$$

(Notice that the term $2(1-\delta)$ in $(2.5)$ and $(2.6)$ is replaced now with $1-\delta$.)

To prove that (2.5) holds we follow the computations given in (2.12) through (2.16) to get

$$
m A_{1}(\varphi)=2 m \sum_{\lambda \in \Lambda}\left(\bar{\delta}_{\lambda} \varphi\right) L_{\lambda}^{0} T_{\lambda} \varphi=2 m I_{1}+2 m I_{2}^{(1)}+2 m I_{2}^{(2)}
$$

where $2 m I_{1}$ is the expression on the left-hand side of (4.4), and $I_{2}^{(j)}$ are given by (2.15) and (2.16). Observe that, for any unit $\xi \in \mathbb{R}^{d}$ and $q_{\lambda(\xi)}:=\xi_{i} D_{i} q_{\lambda}$ we have $\left|q_{\lambda(\xi)}\right| \leq 2 C r_{\lambda}$ where $C$ is the Lipschitz constant of $r_{\lambda}$. Thus due to (4.3) and the assumption that $q_{\mu} \leq \theta \chi_{\mu}$ we have

$$
\left(\sum_{\mu \in \Lambda_{1}} q_{\mu(\xi)} \Delta_{\mu} \varphi\right)^{2} \leq N\left(\sum_{\mu \in \Lambda_{1}} \sqrt{q_{\mu} \tau_{\mu}}\left|\Delta_{\mu} \varphi\right|\right)^{2} \leq N \sum_{\mu \in \Lambda_{1}} \mathcal{Q}\left(\bar{\delta}_{\mu} \varphi\right)
$$

where $N$ is a generic constant depending on $\bar{\tau}, \theta$, the Lipschitz constants of $r_{\lambda}$ and on the number of vectors in $\Lambda_{1}$. Furthermore,

$$
\left(\sum_{\mu \in \Lambda_{1}} p_{\mu(\xi)} \delta_{\mu} \varphi\right)^{2} \leq N\left(\sum_{\mu \in \Lambda_{1}}\left|\bar{\delta}_{\mu} \varphi\right|\right)^{2} \leq N \sum_{\mu \in \Lambda_{1}}\left|\bar{\delta}_{\mu} \varphi\right|^{2},
$$

where $\xi:=D \varphi /|D \varphi|$ and $N$ is a constant depending only on the Lipschitz constants of $p_{\mu}, \bar{\tau}$, and and on the number of vectors in $\Lambda_{1}$. Using these inequalities we obtain

$$
\begin{gathered}
2 m I_{2}^{(1)} \leq m \tau_{0}^{3}|D \varphi|^{2}+\tau_{0} m\left(\sum_{\mu \in \Lambda_{1}} q_{\mu(\xi)} \Delta_{\mu} \varphi\right)^{2} \\
\leq c m \tau_{0} c_{0}^{-1} \sum_{\mu \in \Lambda_{2}}\left|\bar{\delta}_{\mu} \varphi\right|^{2}+\tau_{0} m N \sum_{\mu \in \Lambda_{1}} \mathcal{Q}\left(\bar{\delta}_{\mu} \varphi\right), \\
m I_{2}^{(2)} \leq m \tau_{0}^{3}|D \varphi|^{2}+\tau_{0} m\left(\sum_{\mu \in \Lambda_{1}} p_{\mu(\xi)} \Delta_{\mu} \varphi\right)^{2} \\
\leq c \tau_{0} m c_{0}^{-1} N \sum_{\lambda \in \Lambda}\left|\bar{\delta}_{\lambda} \varphi\right|^{2}
\end{gathered}
$$


with a constant $N$ depending on $\bar{\tau}, \theta$, the Lipschitz constants of $r_{\lambda}, p_{\lambda}$ and on the number of vectors in $\Lambda_{1}$. Taking here $\tau_{0}>0$ sufficiently small we get

$$
2 m I_{2}^{(1)}+2 m I_{2}^{(2)} \leq \frac{\delta}{2} \sum_{\mu \in \Lambda_{1}} \mathcal{Q}\left(\bar{\delta}_{\mu} \varphi\right)+\frac{\delta}{2} c \sum_{\lambda \in \Lambda}\left|\bar{\delta}_{\lambda} \varphi\right|^{2} .
$$

Adding this inequality to inequality (4.4) we see that there exist constants $\delta, \bar{\tau}_{0} \in(0,1]$ ( $\delta$ can be taken half of the one in (4.3)), such that

$$
m A_{1}(\varphi) \leq(1-\delta) \sum_{\lambda \in \Lambda} \mathcal{Q}\left(\bar{\delta}_{\lambda} \varphi\right)+K_{1} \mathcal{Q}(\varphi)+(1-\delta) c \mathcal{K}\left(\sum_{\lambda \in \Lambda}\left|\bar{\delta}_{\lambda} \varphi\right|^{2}\right)
$$

on $H_{T}$ for all smooth functions $\varphi$ provided that $\tau_{0} \in\left(0, \bar{\tau}_{0}\right]$. Thus, a condition even somewhat stronger than (2.5) is satisfied. We note that by using Remarks 5.1 and 5.2 of [14], one can also see that due to (4.4) and the above mentioned properties of $r_{\lambda}$ and $p_{\lambda}$ and due to (4.3), condition (4.7) holds.

Next, observe that the left-hand side of (2.6) equals

$$
B+A^{\prime}+A_{q}^{\prime \prime}+A_{p}^{\prime \prime}+B^{\prime}+B^{\prime \prime},
$$

where $B$ is the left-hand side of (4.5),

$$
\begin{gathered}
A^{\prime}=n \sum_{\nu \in \Lambda_{2}} A_{1}\left(\bar{\delta}_{\nu} \varphi\right), \quad A_{q}^{\prime \prime}=2 n \sum_{\lambda \in \Lambda_{1}, \nu \in \Lambda_{2}}\left(\bar{\delta}_{\nu} \bar{\delta}_{\lambda} \varphi\right) Q_{\nu} \bar{\delta}_{\lambda} \varphi \\
A_{p}^{\prime \prime}=2 n \sum_{\lambda \in \Lambda_{1}, \nu \in \Lambda_{2}}\left(\bar{\delta}_{\nu} \bar{\delta}_{\lambda} \varphi\right) P_{\nu} \bar{\delta}_{\lambda} \varphi \\
B^{\prime}=2 n(n-1) \sum_{\lambda \in \Lambda_{1} \times \Lambda_{2}}\left(\bar{\delta}_{\lambda} \varphi\right) Q_{\lambda} T_{\lambda} \varphi \\
B^{\prime \prime}=n(n-1) \sum_{\lambda \in \Lambda_{2}^{2}}\left(\bar{\delta}_{\lambda} \varphi\right) Q_{\lambda} \varphi .
\end{gathered}
$$

Here by $(4.7)$

$$
\begin{gathered}
A^{\prime} \leq(1-\delta) \sum_{\nu \in \Lambda_{2}, \lambda \in \Lambda} \mathcal{Q}\left(\bar{\delta}_{\lambda} \bar{\delta}_{\nu} \varphi\right)+K_{1} \sum_{\nu \in \Lambda_{2}} \mathcal{Q}\left(\bar{\delta}_{\nu} \varphi\right) \\
+(1-\delta) c \mathcal{K}\left(\sum_{\lambda \in \Lambda^{2}}\left|\bar{\delta}_{\lambda} \varphi\right|^{2}\right) .
\end{gathered}
$$

Then,

$$
\begin{gathered}
A_{q}^{\prime \prime}=2 n \tau_{0}^{2} \sum_{\lambda, \mu \in \Lambda_{1}} \sum_{j=1}^{d}\left(D_{j} \bar{\delta}_{\lambda} \varphi\right)\left(D_{j} r_{\mu}\right)\left[r_{\mu} \Delta_{\mu} \bar{\delta}_{\lambda} \varphi\right] \\
\leq(1 / 16) \delta c \tau_{0}^{2} \sum_{\lambda \in \Lambda_{1}} \sum_{j=1}^{d}\left(D_{j} \bar{\delta}_{\lambda} \varphi\right)^{2}+N \tau_{0}^{2} \sum_{\mu \in \Lambda_{1}, \lambda \in \Lambda_{1}^{2}} q_{\mu}\left(\bar{\delta}_{\mu} \bar{\delta}_{\lambda} \varphi\right)^{2}
\end{gathered}
$$




$$
\begin{gathered}
=(1 / 16) \delta c \sum_{\lambda \in \Lambda_{1}, \nu \in \Lambda_{2}}\left(\bar{\delta}_{\nu} \bar{\delta}_{\lambda} \varphi\right)^{2}+N \tau_{0}^{2} \sum_{\mu \in \Lambda_{1}, \lambda \in \Lambda_{1}^{2}} q_{\mu}\left(\bar{\delta}_{\mu} \bar{\delta}_{\lambda} \varphi\right)^{2} \\
\leq(1 / 16) \delta c \sum_{\lambda \in \Lambda^{2}}\left(\bar{\delta}_{\lambda} \varphi\right)^{2}+N \tau_{0}^{2} \sum_{\lambda \in \Lambda^{2}} \mathcal{Q}\left(\bar{\delta}_{\lambda} \varphi\right),
\end{gathered}
$$

where and below by $N$ we denote various generic constants independent of $\varphi,(t, x)$, and $\tau_{0}$. Next, quite similarly

$$
\begin{gathered}
A_{p}^{\prime \prime}=2 n \tau_{0}^{2} \sum_{\lambda, \mu \in \Lambda_{1}} \sum_{j=1}^{d}\left(D_{j} \bar{\delta}_{\lambda} \varphi\right)\left(D_{j} p_{\mu}\right) \bar{\delta}_{\mu} \bar{\delta}_{\lambda} \varphi \\
\leq(1 / 16) \delta c \tau_{0}^{2} \sum_{\lambda \in \Lambda_{1}} \sum_{j=1}^{d}\left(D_{j} \bar{\delta}_{\lambda} \varphi\right)^{2}+N \tau_{0}^{2} \sum_{\lambda \in \Lambda_{1}^{2}}\left(\bar{\delta}_{\lambda} \varphi\right)^{2} \\
\leq(1 / 16) \delta c \sum_{\lambda \in \Lambda^{2}}\left(\bar{\delta}_{\lambda} \varphi\right)^{2}+N \tau_{0}^{2} \sum_{\lambda \in \Lambda^{2}}\left(\bar{\delta}_{\lambda} \varphi\right)^{2} .
\end{gathered}
$$

Now we estimate $B^{\prime}$ and $B^{\prime \prime}$. We have

$$
B^{\prime}=n(n-1) \tau_{0}^{2} \sum_{\lambda, \mu \in \Lambda_{1}} \sum_{j=1}^{d}\left(D_{j} \bar{\delta}_{\lambda} \varphi\right)\left(D_{j} \bar{\delta}_{\lambda} q_{\mu}\right) \Delta_{\mu} T_{\lambda} \varphi .
$$

Here

$$
\Delta_{\mu} T_{\lambda}=\Delta_{\mu}+\left(\delta_{\mu}+\delta_{-\mu}\right) \delta_{\lambda}
$$

and it is seen that

$$
\begin{aligned}
& B^{\prime} \leq(1 / 16) \delta c \tau_{0}^{2} \sum_{\lambda \in \Lambda_{1}} \sum_{j=1}^{d}\left(D_{j} \bar{\delta}_{\lambda} \varphi\right)^{2}+N \tau_{0}^{2} \sum_{\lambda \in \Lambda^{2}}\left(\bar{\delta}_{\lambda} \varphi\right)^{2} \\
& \leq(1 / 16) \delta c \sum_{\lambda \in \Lambda^{2}}\left(\bar{\delta}_{\lambda} \varphi\right)^{2}+N \tau_{0}^{2} \sum_{\lambda \in \Lambda^{2}}\left(\bar{\delta}_{\lambda} \varphi\right)^{2}
\end{aligned}
$$

Similarly,

$$
\begin{aligned}
B^{\prime \prime}= & (1 / 2) n(n-1) \tau_{0}^{4} \sum_{j, k=1}^{d} \sum_{\mu \in \Lambda_{1}}\left(D_{j k} \varphi\right)\left(D_{j k} q_{\mu}\right) \Delta_{\mu} \varphi \\
& \leq(1 / 16) \delta c \sum_{\lambda \in \Lambda^{2}}\left(\bar{\delta}_{\lambda} \varphi\right)^{2}+N \tau_{0}^{4} \sum_{\lambda \in \Lambda^{2}}\left(\bar{\delta}_{\lambda} \varphi\right)^{2} .
\end{aligned}
$$

By combining the above estimates we see that the left-hand side of (2.6) is majorated by

$$
\left(1-\delta+N \tau_{0}^{2}\right) \sum_{\nu \in \Lambda^{2}} \mathcal{Q}\left(\bar{\delta}_{\nu} \varphi\right)+K_{1} \sum_{\nu \in \Lambda} \mathcal{Q}\left(\bar{\delta}_{\nu} \varphi\right)+K_{1} \mathcal{K}\left(\sum_{\lambda \in \Lambda}\left|\bar{\delta}_{\lambda} \varphi\right|^{2}\right)
$$




$$
+c\left[2(1-\delta) \mathcal{K}\left(\sum_{\lambda \in \Lambda^{2}}\left|\bar{\delta}_{\lambda} \varphi\right|^{2}\right)+\left((1 / 4) \delta+N \tau_{0}^{2}\right) \sum_{\lambda \in \Lambda^{2}}\left|\bar{\delta}_{\lambda} \varphi\right|^{2}\right] .
$$

It follows easily that by choosing $\tau_{0}$ small enough we will satisfy (2.6) as well as (2.5) with $\delta / 2$ in place of $\delta$ and appropriate $K_{1}$.

Remark 4.3. In [14] we have seen that even Assumption 2.4 imposes certain nontrivial structural conditions on $q_{\lambda}$ which cannot be guaranteed by the size of $c_{0}$ if $q_{\lambda}$ is only once continuously differentiable.

In contrast, given that Assumptions 2.1, 2.2, 2.4 are satisfied and $m \geq 2$, we claim that Assumption 2.5 is also satisfied if $c_{0}$ is large enough.

To prove our claim we notice that by $(3.5)$

$$
\sum_{\lambda \in \Lambda^{2}}\left|Q_{\lambda} T_{\lambda} \varphi\right|^{2} \leq N \mathcal{K}\left(\sum_{\mu \in \Lambda^{2}}\left|\bar{\delta}_{\mu} \varphi\right|^{2}\right),
$$

so that

$$
\begin{gathered}
n(n-1) \sum_{\lambda \in \Lambda^{2}}\left(\bar{\delta}_{\lambda} \varphi\right) Q_{\lambda} T_{\lambda} \varphi \\
\leq m(m-1)\left[\sum_{\lambda \in \Lambda^{2}}\left|\bar{\delta}_{\lambda} \varphi\right|^{2}+N \mathcal{K}\left(\sum_{\mu \in \Lambda^{2}}\left|\bar{\delta}_{\mu} \varphi\right|^{2}\right)\right]=: N^{\prime} \mathcal{K}\left(\sum_{\mu \in \Lambda^{2}}\left|\bar{\delta}_{\mu} \varphi\right|^{2}\right) .
\end{gathered}
$$

Now assume that $c$ is so large that

$$
N^{\prime} \leq \delta c .
$$

Then it follows from (2.5) that the left-hand side of (2.6) is majorated by

$$
(1-\delta) \sum_{\lambda \in \Lambda_{1}, \nu \in \Lambda^{2}} \chi_{\lambda}\left|\delta_{\lambda} \bar{\delta}_{\nu} \varphi\right|^{2}+K_{1} \sum_{\lambda \in \Lambda_{1}, \nu \in \Lambda} \chi_{\lambda}\left|\delta_{\lambda} \bar{\delta}_{\nu} \varphi\right|^{2}+I
$$

where

$$
\begin{gathered}
I=2(1-\delta) c \mathcal{K}\left(\sum_{\lambda \in \Lambda^{2}}\left|\bar{\delta}_{\lambda} \varphi\right|^{2}\right)+\delta c \mathcal{K}\left(\sum_{\mu \in \Lambda^{2}}\left|\bar{\delta}_{\mu} \varphi\right|^{2}\right) \\
=: 2(1-\delta / 2) c \mathcal{K}\left(\sum_{\lambda \in \Lambda^{2}}\left|\bar{\delta}_{\lambda} \varphi\right|^{2}\right) .
\end{gathered}
$$

We thus obtain (2.6) with $\delta / 2$ in place of $\delta$.

REMARK 4.4. It is interesting to have sufficiently simple conditions on the coefficients of differential operators $\mathcal{L}$ which guarantee that there exist finite-difference schemes for which our assumptions hold. Here we will only give a one dimensional example. This example is based on the results of Remark 4.5 below, which can also be used to analyze many multi-dimensional situations as well in the spirit of the comments in [14].

Take $d=1$ and

$$
\mathcal{L} \varphi(x)=a(x) \varphi^{\prime \prime}(x)+b(x) \varphi^{\prime}(x)-c(x) \varphi(x) .
$$


We assume that $a \geq 0$ and $r:=\sqrt{a}, b$, and $c$ are $m$-times continuously differentiable with bounded derivatives. We take $\Lambda_{1}=\{ \pm 1\}$ and define

$$
q_{\mu}=a, \quad p_{\mu}=(1 / 2) \mu b+\theta,
$$

where $\theta$ is a constant such that $p_{\mu} \geq 1$. By using an argument in Remark 6.7 of [14] and using our Remark 4.5, one can easily derive that, for a sufficiently small $\tau_{0}$ and $\tau_{\mu} \equiv 1$, Assumptions 2.4 and 2.5 are satisfied for all sufficiently small $h$ (with perhaps different $\delta$ and $\left.K_{1}\right)$ if, for $n \leq m$,

$$
75 n^{2}\left(r^{\prime}\right)^{2}+2 n b^{\prime} \leq(1-\delta) c+K_{1} a
$$

(cf. (4.2)). Again as in [14] we see that at points where $a$ is close to zero either $c$ should be large or $b^{\prime}$ be sufficiently negative.

REMARK 4.5. Condition (4.4) and its implications are discussed in many details in [14] (with $2 c$ in place of $c$ ). Here we give sufficient conditions for (4.4) and (4.5) to be satisfied without involving test functions $\varphi$. For simplicity, we only do it in case

$$
\tau_{\lambda}=1 \text { for all } \lambda \in \Lambda_{1} .
$$

It is obvious that if we define $\xi_{\lambda \mu}=\delta_{\lambda} \delta_{\mu} \varphi$, then condition (4.5) can be rewritten in terms of $\xi_{\lambda \mu}$. What is nontrivial is that one can give sufficient conditions for (4.5) to hold in terms of $\xi_{\lambda}$ and not the two-parameter object $\xi_{\lambda \mu}$. In addition, we will see that these sufficient conditions are obtained just by slightly strengthening the corresponding conditions from [14] guaranteeing the first-order derivatives estimates. As in [14] one could extract further implications and simplifications of the new conditions of the type that on the set where $c$ is small we need $\chi_{\lambda}$ to be uniformly bounded away from zero or $p_{\lambda}$ be sufficiently strongly monotone (see [14] for more details).

As in Remark 4.2 we assume that $\Lambda_{1}=-\Lambda_{1}, q_{\lambda}=q_{-\lambda}(\geq 0)$ and, for a constant $\theta>0$, we have $q_{\lambda} \leq \theta \chi_{\lambda}$ for all $h \in\left(0, h_{0}\right]$ and $\lambda \in \Lambda_{1}$. Moreover, we assume additionally that $r_{\lambda}:=\sqrt{q_{\lambda}}$ is twice continuously differentiable in $x$ and is bounded on $H_{T}$ along with first and second-order derivatives in $x$. Also we fix a constant $\delta \in(0,1 / 4]$ and assume that on $H_{T}$ there are functions $r_{\lambda \mu}=r_{h \lambda \mu}, p_{\lambda \mu}=p_{h \lambda \mu} \geq 0$, $\lambda, \mu \in \Lambda_{1}$, such that

$$
\begin{gathered}
m(m-1) h^{2}\left(\delta_{\lambda} r_{\mu}\right)^{2} \leq \delta\left(\chi_{\lambda}+\chi_{\mu}\right)+h^{2} r_{\lambda \mu}^{2}, \quad \sum_{\mu \in \Lambda_{1}} \sup _{\lambda \in \Lambda_{1}} r_{\lambda \mu}^{2} \leq \delta c \\
h^{2}\left|\delta_{\lambda} p_{\mu}\right| \leq \delta^{2}\left(\chi_{\lambda}+\chi_{\mu}\right)+\delta h^{2} p_{\lambda \mu}, \quad \sum_{\mu \in \Lambda_{1}} \sup _{\lambda \in \Lambda_{1}} p_{\lambda \mu} \leq \delta c .
\end{gathered}
$$

By virtue of Remark 6.1 of [14] one can always find approximations $L_{h}^{\prime}$ of the zero operator such that $L_{h}+L_{h}^{\prime}$ will still be approximating $\mathcal{L}$ and for the coefficients $p_{\lambda}^{\prime}$ of $L_{h}+L_{h}^{\prime}$ we will have $p_{\lambda}^{\prime} \geq 1$. Obviously, for $L_{h}+L_{h}^{\prime}$ conditions (4.8) and (4.9) are satisfied with $r_{\lambda \mu}=p_{\lambda \mu}=0$ for sufficiently small $h$.

For a function $\xi_{\lambda}$ given on $\Lambda_{1}$ let us write

$$
|\xi|^{2}=\sum_{\lambda \in \Lambda_{1}}\left|\xi_{\lambda}\right|^{2}
$$

and let us drop the summation sign over repeated indices in $\Lambda_{1}$. Then we claim that 
Conditions (4.4) and (4.5) are satisfied with appropriate $K_{1}, \mathcal{K}$, and $\delta$ if on $H_{T}$ for all functions $\xi_{\lambda}$ and $n=1, \ldots, m$ we have

$$
\begin{gathered}
28 n^{2}(1-4 \delta)^{-1} J_{1}+(9 / 2) n^{2}(1-4 \delta)^{-1} J_{2}+(1 / 2) n^{2} J_{3} \\
+2 \delta n^{2} \sum_{\lambda, \mu \in \Lambda_{1}} \xi_{\lambda}^{2}\left|\delta_{\lambda} p_{\mu}\right|+2 n \xi_{\lambda} \xi_{\mu}\left(\delta_{\lambda} p_{\mu}+\left(\delta_{\lambda} r_{\mu}\right)^{2}\right) \\
\leq(1-4 \delta) c|\xi|^{2}+K_{1} \xi_{\lambda}^{2} \chi_{\lambda}+\delta h^{-2} \chi_{\lambda}\left|\xi_{\lambda}+\xi_{-\lambda}\right|^{2}
\end{gathered}
$$

where

$$
J_{1}=\sum_{\mu, \lambda \in \Lambda_{1}} \xi_{\lambda}^{2}\left(\delta_{\lambda} r_{\mu}\right)^{2}, \quad J_{2}=\sum_{\mu \in \Lambda_{1}}\left(\sum_{\lambda \in \Lambda_{1}} \xi_{\lambda} \delta_{\lambda} r_{\mu}\right)^{2}, \quad J_{3}=\sum_{\lambda, \mu \in \Lambda_{1}}\left(\delta_{\lambda} r_{\mu}\right)^{2} \xi_{\mu}^{2} .
$$

To prove this claim, introduce

$$
\begin{gathered}
J_{1}(\varphi)=\sum_{\mu, \lambda \in \Lambda_{1}}\left(\delta_{\lambda} \varphi\right)^{2}\left(\delta_{\lambda} r_{\mu}\right)^{2} \\
J_{2}(\varphi)=\sum_{\mu \in \Lambda_{1}}\left(\sum_{\lambda \in \Lambda_{1}}\left(\delta_{\lambda} \varphi\right) \delta_{\lambda} r_{\mu}\right)^{2}, \quad J_{3}(\varphi)=\sum_{\lambda, \mu \in \Lambda_{1}}\left(\delta_{\mu} \varphi\right)^{2}\left(\delta_{\lambda} r_{\mu}\right)^{2}
\end{gathered}
$$

and first recall that by Remarks 6.2 and 6.3 of [14] after replacing there $c$ with $c / 2$ we obtain

$$
\begin{aligned}
& 2 n \sum_{\lambda \in \Lambda_{1}}\left(\delta_{\lambda} \varphi\right) L_{\lambda}^{0} T_{\lambda} \varphi+18 n^{2} J_{1}(\varphi)+(5 / 2) n^{2} J_{2}(\varphi)+(1 / 2) n^{2} J_{3}(\varphi) \\
& \quad \leq(1-\delta) \sum_{\lambda \in \Lambda_{1}} \mathcal{Q}\left(\delta_{\lambda} \varphi\right)+K_{1} \mathcal{Q}(\varphi)+(1-\delta) c \mathcal{K}\left(\sum_{\lambda \in \Lambda_{1}}\left|\delta_{\lambda} \varphi\right|^{2}\right) .
\end{aligned}
$$

In particular condition (4.4) is satisfied. Furthermore, by substituting $\delta_{\nu} \varphi$ in place of $\varphi$ and summing up over $\nu \in \Lambda_{1}$, we get

$$
\begin{gathered}
2 n \sum_{\lambda, \nu \in \Lambda_{1}}\left(\delta_{\nu} \delta_{\lambda} \varphi\right) L_{\lambda}^{0} T_{\lambda} \delta_{\nu} \varphi+18 n^{2} \sum_{\nu \in \Lambda_{1}} J_{1}\left(\delta_{\nu} \varphi\right) \\
+(5 / 2) n^{2} \sum_{\nu \in \Lambda_{1}} J_{2}\left(\delta_{\nu} \varphi\right)+(1 / 2) n^{2} \sum_{\nu \in \Lambda_{1}} J_{3}\left(\delta_{\nu} \varphi\right) \\
\leq(1-\delta) \sum_{\lambda \in \Lambda_{1}^{2}} \mathcal{Q}\left(\delta_{\lambda} \varphi\right)+K_{1} \sum_{\nu \in \Lambda_{1}} \mathcal{Q}\left(\delta_{\nu} \varphi\right)+(1-\delta) c \mathcal{K}\left(\sum_{\lambda \in \Lambda_{1}^{2}}\left|\delta_{\lambda} \varphi\right|^{2}\right) .
\end{gathered}
$$

It follows that to prove our claim, it suffices to prove that

$$
n(n-1) \sum_{\lambda \in \Lambda_{1}^{2}}\left(\delta_{\lambda} \varphi\right) Q_{\lambda} T_{\lambda} \varphi \leq 18 n^{2} \sum_{\nu \in \Lambda_{1}} J_{1}\left(\delta_{\nu} \varphi\right)+(5 / 2) n^{2} \sum_{\nu \in \Lambda_{1}} J_{2}\left(\delta_{\nu} \varphi\right)
$$




$$
\begin{gathered}
+(1 / 2) n^{2} \sum_{\nu \in \Lambda_{1}} J_{3}\left(\delta_{\nu} \varphi\right)+(2 / 3) \delta \sum_{\nu \in \Lambda_{1}^{2}} \mathcal{Q}\left(\delta_{\nu} \varphi\right) \\
+(1 / 3) \delta c \mathcal{K}\left(\sum_{\nu \in \Lambda_{1}^{2}}\left|\delta_{\nu} \varphi\right|^{2}\right)+N \sum_{\nu \in \Lambda_{1}} \mathcal{Q}\left(\delta_{\nu} \varphi\right)+N \mathcal{K}\left(\sum_{\nu \in \Lambda_{1}}\left|\delta_{\nu} \varphi\right|^{2}\right),
\end{gathered}
$$

where and below by $N$ we denote generic constants independent of $\varphi$ and $(t, x)$ (and various $\varepsilon$ 's once they appear). Observe that for $\lambda=\left(\lambda^{1}, \lambda^{2}\right) \in \Lambda_{1}^{2}$ and $\mu \in \Lambda_{1}$,

$$
T_{\lambda}=1+h^{2} \delta_{\lambda^{1}} \delta_{\lambda^{2}}+h\left(\delta_{\lambda^{1}}+\delta_{\lambda^{2}}\right), \quad \Delta_{\mu}=h^{-1}\left(\delta_{\mu}+\delta_{-\mu}\right)
$$

and hence

$$
\Delta_{\mu} T_{\lambda}=\Delta_{\mu}+h \delta_{\lambda^{1}} \delta_{\lambda^{2}}\left(\delta_{\mu}+\delta_{-\mu}\right)+\left(\delta_{\lambda^{1}}+\delta_{\lambda^{2}}\right)\left(\delta_{\mu}+\delta_{-\mu}\right),
$$

implying that

$$
\sum_{\lambda \in \Lambda_{1}^{2}}\left(\delta_{\lambda} \varphi\right) Q_{\lambda} T_{\lambda} \varphi=S_{1}+S_{2}
$$

where

$$
\begin{gathered}
S_{1}=(1 / 2) \sum_{\lambda \in \Lambda_{1}^{2}, \mu \in \Lambda_{1}}\left(\delta_{\lambda} \varphi\right)\left(\delta_{\lambda} q_{\mu}\right)\left(4 \delta_{\lambda^{1}}-\delta_{-\mu}\right) \delta_{\mu} \varphi, \\
S_{2}=h \sum_{\lambda \in \Lambda_{1}^{2}, \mu \in \Lambda_{1}}\left(\delta_{\lambda} \varphi\right)\left(\delta_{\lambda} q_{\mu}\right) \delta_{\lambda} \delta_{\mu} \varphi .
\end{gathered}
$$

Next, as it is easy to see for $\lambda \in \Lambda_{1}^{2}$

$$
\begin{gathered}
\delta_{\lambda} q_{\mu}=2\left(\delta_{\lambda^{1}} r_{\mu}\right) \delta_{\lambda^{2}} r_{\mu}+2 r_{\mu} \delta_{\lambda} r_{\mu} \\
+2 h\left(\delta_{\lambda^{1}} r_{\mu}+\delta_{\lambda^{2}} r_{\mu}\right) \delta_{\lambda} r_{\mu}+h^{2}\left(\delta_{\lambda} r_{\mu}\right)^{2} .
\end{gathered}
$$

Estimating $S_{2}$. First we estimate the term $S_{2}$, which contains the third-order differences of $\varphi$. For the main term in $S_{2}$ we have

$$
\begin{gathered}
B_{1}:=2 h \sum_{\lambda \in \Lambda_{1}^{2}}\left(\delta_{\lambda} \varphi\right)\left(\delta_{\lambda^{1}} r_{\mu}\right)\left(\delta_{\lambda^{2}} r_{\mu}\right) \delta_{\lambda} \delta_{\mu} \varphi \\
\leq 16 \sum_{\lambda, \mu, \nu \in \Lambda_{1}}\left(\delta_{\lambda} \delta_{\nu} \varphi\right)^{2}\left(\delta_{\lambda} r_{\mu}\right)^{2}+(1 / 16) h^{2} \sum_{\lambda, \mu, \nu \in \Lambda_{1}}\left(\delta_{\lambda} r_{\mu}\right)^{2}\left(\delta_{\lambda} \delta_{\mu} \delta_{\nu} \varphi\right)^{2} \\
=: 16 \sum_{\nu \in \Lambda_{1}} J_{1}\left(\delta_{\nu} \varphi\right)+(1 / 16) E
\end{gathered}
$$

where by assumption (4.8) and Lemma 6.1 of [14]

$$
n(n-1) E \leq 2 \delta \sum_{\nu \in \Lambda_{1}^{2}} \mathcal{Q}\left(\delta_{\nu} \varphi\right)+4 \delta c \mathcal{K}\left(\sum_{\lambda \in \Lambda_{1}^{2}}\left(\delta_{\lambda} \varphi\right)^{2}\right) .
$$


Hence,

$$
\begin{gathered}
n(n-1) B_{1} \leq 16 n^{2} \sum_{\nu \in \Lambda_{1}} J_{1}\left(\delta_{\nu} \varphi\right) \\
+(1 / 8) \delta \sum_{\nu \in \Lambda_{1}^{2}} \mathcal{Q}\left(\delta_{\nu} \varphi\right)+(1 / 4) \delta c \mathcal{K}\left(\sum_{\lambda \in \Lambda_{1}^{2}}\left(\delta_{\lambda} \varphi\right)^{2}\right) .
\end{gathered}
$$

Next, obviously, for any $\varepsilon>0$, (here we use that $q_{\lambda} \leq \theta \chi_{\lambda}$ )

$$
\begin{gathered}
B_{2}:=2 h \sum_{\lambda \in \Lambda_{1}^{2}, \mu \in \Lambda_{1}}\left(\delta_{\lambda} \varphi\right) r_{\mu}\left(\delta_{\lambda} r_{\mu}\right) \delta_{\lambda} \delta_{\mu} \varphi \\
\leq \varepsilon^{-1} h^{2} \sum_{\lambda \in \Lambda_{1}^{2}, \mu \in \Lambda_{1}}\left(\delta_{\lambda} \varphi\right)^{2}\left(\delta_{\lambda} r_{\mu}\right)^{2}+\varepsilon \sum_{\lambda \in \Lambda_{1}^{2}, \mu \in \Lambda_{1}} q_{\mu}\left(\delta_{\lambda} \delta_{\mu} \varphi\right)^{2} \\
\leq N \varepsilon^{-1} \mathcal{K}\left(\sum_{\lambda \in \Lambda_{1}}\left(\delta_{\lambda} \varphi\right)^{2}\right)+\varepsilon \sum_{\nu \in \Lambda_{1}^{2}} \mathcal{Q}\left(\delta_{\nu} \varphi\right) .
\end{gathered}
$$

It follows that (with $\varepsilon>0$ different from the one from above but still arbitrary)

$$
n(n-1) B_{2} \leq N \varepsilon^{-1} \mathcal{K}\left(\sum_{\lambda \in \Lambda_{1}}\left(\delta_{\lambda} \varphi\right)^{2}\right)+\varepsilon \sum_{\nu \in \Lambda_{1}^{2}} \mathcal{Q}\left(\delta_{\nu} \varphi\right) .
$$

Also

$$
\begin{gathered}
n(n-1) h^{2} \sum_{\lambda \in \Lambda_{1}^{2}, \mu \in \Lambda_{1}}\left|\left(\delta_{\lambda} \varphi\right) \delta_{\lambda} \delta_{\mu} \varphi\right| \\
\leq \varepsilon \delta c \sum_{\lambda \in \Lambda_{1}^{2}}\left(\delta_{\lambda} \varphi\right)^{2}+N \varepsilon^{-1} \mathcal{K}\left(\sum_{\lambda \in \Lambda_{1}}\left(\delta_{\lambda} \varphi\right)^{2}\right) .
\end{gathered}
$$

Upon combining this with (4.12) and (4.13) we obtain

$$
\begin{aligned}
& n(n-1) S_{2} \leq(\varepsilon+\delta / 8) \sum_{\nu \in \Lambda_{1}^{2}} \mathcal{Q}\left(\delta_{\nu} \varphi\right)+16 n^{2} \sum_{\nu \in \Lambda_{1}} J_{1}\left(\delta_{\nu} \varphi\right) \\
& \quad+(\varepsilon+1 / 4) \delta c \mathcal{K}\left(\sum_{\lambda \in \Lambda_{1}^{2}}\left(\delta_{\lambda} \varphi\right)^{2}\right)+N \varepsilon^{-1} \mathcal{K}\left(\sum_{\lambda \in \Lambda_{1}}\left(\delta_{\lambda} \varphi\right)^{2}\right) .
\end{aligned}
$$

Estimating $S_{1}$. We again start with the main term in $S_{1}$, which we split into two parts writing

$$
\left(4 \delta_{\lambda^{1}}-\delta_{-\mu}\right) \delta_{\mu} \varphi=4 \delta_{\lambda^{1}} \delta_{\mu} \varphi+\Delta_{\mu} \varphi
$$

We have

$$
4 \sum_{\lambda \in \Lambda_{1}^{2}, \mu \in \Lambda_{1}}\left(\delta_{\lambda} \varphi\right)\left(\delta_{\lambda^{2}} r_{\mu}\right)\left(\delta_{\lambda^{1}} r_{\mu}\right) \delta_{\lambda^{1}} \delta_{\mu} \varphi
$$




$$
\begin{gathered}
=4 \sum_{\nu, \mu \in \Lambda_{1}}\left[\sum_{\lambda \in \Lambda_{1}}\left(\delta_{\lambda}\left(\delta_{\nu} \varphi\right)\right) \delta_{\lambda} r_{\mu}\right]\left(\delta_{\nu} r_{\mu}\right) \delta_{\nu} \delta_{\mu} \varphi \\
\leq 2 \sum_{\nu, \mu \in \Lambda_{1}}\left[\sum_{\lambda \in \Lambda_{1}}\left(\delta_{\lambda} \delta_{\nu} \varphi\right) \delta_{\lambda} r_{\mu}\right]^{2}+2 \sum_{\nu, \mu \in \Lambda_{1}}\left(\delta_{\nu} r_{\mu}\right)^{2}\left(\delta_{\nu} \delta_{\mu} \varphi\right)^{2} \\
\leq 2 \sum_{\nu \in \Lambda_{1}} J_{2}\left(\delta_{\nu} \varphi\right)+2 \sum_{\nu, \mu, \lambda \in \Lambda_{1}}\left(\delta_{\nu} r_{\mu}\right)^{2}\left(\delta_{\nu} \delta_{\lambda} \varphi\right)^{2} \\
=2 \sum_{\nu \in \Lambda_{1}} J_{2}\left(\delta_{\nu} \varphi\right)+2 \sum_{\lambda \in \Lambda_{1}} J_{1}\left(\delta_{\lambda} \varphi\right)
\end{gathered}
$$

Furthermore,

$$
\begin{gathered}
\sum_{\lambda \in \Lambda_{1}^{2}, \mu \in \Lambda_{1}}\left(\delta_{\lambda} \varphi\right)\left(\delta_{\lambda^{1}} r_{\mu}\right)\left(\delta_{\lambda^{2}} r_{\mu}\right) \Delta_{\mu} \varphi \\
=\sum_{\nu, \mu \in \Lambda_{1}}\left[\sum_{\lambda \in \Lambda_{1}}\left(\delta_{\lambda} \delta_{\nu} \varphi\right) \delta_{\lambda} r_{\mu}\right]\left(\delta_{\nu} r_{\mu}\right) \Delta_{\mu} \varphi \\
\leq(1 / 2) \sum_{\nu, \mu \in \Lambda_{1}}\left[\sum_{\lambda \in \Lambda_{1}}\left(\delta_{\lambda} \delta_{\nu} \varphi\right) \delta_{\lambda} r_{\mu}\right]^{2}+(1 / 2) \sum_{\nu, \mu \in \Lambda_{1}}\left(\delta_{\nu} r_{\mu}\right)^{2}\left(\Delta_{\mu} \varphi\right)^{2} \\
\leq(1 / 2) \sum_{\nu \in \Lambda_{1}} J_{2}\left(\delta_{\nu} \varphi\right)+(1 / 2) \sum_{\nu \in \Lambda_{1}} J_{3}\left(\delta_{\nu} \varphi\right) .
\end{gathered}
$$

Next, obviously

$$
\begin{gathered}
4 \sum_{\lambda \in \Lambda_{1}^{2} \mu \in \Lambda_{1}}\left[\left(\delta_{\lambda} \varphi\right)\left(\delta_{\lambda} r_{\mu}\right)\right] r_{\mu} \delta_{\lambda^{1}} \delta_{\mu} \varphi \leq \varepsilon c \delta \sum_{\lambda \in \Lambda_{1}^{2}}\left(\delta_{\lambda} \varphi\right)^{2}+\varepsilon^{-1} N \sum_{\lambda \in \Lambda_{1}} \mathcal{Q}\left(\delta_{\lambda} \varphi\right), \\
\sum_{\lambda \in \Lambda_{1}^{2}}\left[\left(\delta_{\lambda} \varphi\right)\left(\delta_{\lambda} r_{\mu}\right)\right] r_{\mu} \Delta_{\mu} \varphi \leq \varepsilon c \delta \sum_{\lambda \in \Lambda_{1}^{2}}\left(\delta_{\lambda} \varphi\right)^{2}+\varepsilon^{-1} N \sum_{\lambda \in \Lambda_{1}} \mathcal{Q}\left(\delta_{\lambda} \varphi\right) .
\end{gathered}
$$

Finally,

$$
h \sum_{\lambda \in \Lambda_{1}^{2}, \mu \in \Lambda_{1}}\left|\left(\delta_{\lambda} \varphi\right) \delta_{\lambda^{1}} \delta_{\mu} \varphi\right| \leq \varepsilon c \delta \sum_{\lambda \in \Lambda_{1}^{2}}\left(\delta_{\lambda} \varphi\right)^{2}+\varepsilon^{-1} N \mathcal{K}\left(\sum_{\lambda \in \Lambda_{1}}\left(\delta_{\lambda} \varphi\right)^{2}\right) .
$$

Upon combining the above estimates we obtain

$$
\begin{aligned}
& n(n-1) S_{1} \leq n^{2} \sum_{\nu \in \Lambda_{1}}\left[2 J_{1}\left(\delta_{\nu} \varphi\right)+(5 / 2) J_{2}\left(\delta_{\nu} \varphi\right)+(1 / 2) J_{3}\left(\delta_{\nu} \varphi\right)\right] \\
& \quad+\varepsilon c \delta \sum_{\lambda \in \Lambda_{1}^{2}}\left(\delta_{\lambda} \varphi\right)^{2}+\varepsilon^{-1} N\left(\sum_{\lambda \in \Lambda_{1}} \mathcal{Q}\left(\delta_{\lambda} \varphi\right)+\mathcal{K}\left(\sum_{\lambda \in \Lambda_{1}}\left(\delta_{\lambda} \varphi\right)^{2}\right)\right) .
\end{aligned}
$$

This along with (4.14) leads to (4.11) after appropriately choosing $\varepsilon$ and proves our claim. 


\section{REFERENCES}

[1] D.G. ARonson, The stability of finite difference approximations to second order linear parabolic differential equations, Duke Math. J., 30 (1963), pp. 117-128.

[2] G. BARLES AND E.R. JAKOBSEN, On the convergence rate of approximations schemes for Hamilton-Jacobi-Bellman equations, Math. Modelling and Numerical analysis, ESAIM, M2AM, 36:1 (2002), pp. 33-54.

[3] G. BARLes AND E.R. JAKobSEn, Error bounds monotone approximation schemes for HamiltonJacobi-Bellman equations, SIAM J. Numer. Anal., 43:2 (2005), pp. 540-558.

[4] K. BöHmer, Asymptotic expansion for the discretization error in linear elliptic boundary value problems on general regions, Math. Z., 177:2 (1981), pp. 235-255.

[5] L. Collatz, Numerische Behandlung von Differentialgbeichungen, Springer, Berlin (1955).

[6] R. Courant, K. Friedrichs, and H. Lewy, Über die partiellen Differenzengleichungen der mathematischen Physik, Math. Ann., 100:1 (1928), pp. 32-74.

[7] H. Dong AND N.V. KRYlov, On the rate of convergence of finite-difference approximations for Bellman equations with constant coefficients, Algebra i Analiz, 17:2 (2005), pp. 108-132; St. Petersburg Math. J, 17:2 (2006), pp. 295-313.

[8] H. Dong And N.V. KRYlov, On the rate of convergence of finite-difference approximations for degenerate linear parabolic equations with $C^{1}$ and $C^{2}$ coefficients, Electron. J. Diff. Eqns., 2005:102 (2005), pp. 1-25. http://ejde.math.txstate.edu

[9] H. Dong And N.V. KRYlov, On the rate of convergence of finite-difference approximations for parabolic Bellman equations with Lipschitz coefficients in cylindrical domains, Applied Math. and Optimization, 56:1 (2007), pp. 37-66.

[10] J. Douglas JR, The solution of the diffusion equation by a high order correct difference equation, J. Math. Phys., 35 (1956), pp. 145-151.

[11] G.E. Forsythe And W.R. Wasow, Finite Difference Methods for Partial Differential Equations, Wiley, New York (1960).

[12] S. Gerschgorin, Fehlerabschätzung für das Differenzenverfahren zur Lösung partieller Differentialgleichungen, Z. Angew. Math. Mech., 10 (1930), pp. 373-382.

[13] S.K. Godunov and S.V. RYaben'KiI, Difference schemes, An introduction to the underlying theory, (Translated from Russian by E. M. Gelbard), Studies in Mathematics and its Applications, 19. North-Holland Publishing Co., Amsterdam, 1987.

[14] I. Gyöngy and N.V. KRYlov, First derivative estimates for finite difference schemes, Math. Comp, 78 (2009), pp. 2019-2046.

[15] I. GYÖNGY AND N.V. KRYLOV, Expansion of solutions of parameterized equations and acceleration of numerical methods, Illinois J. Math., 50:2 (2006), Special Volume in Memory of Joseph Doob (1910-2004), pp. 473-514.

[16] I. GYÖNGY AND D. SisKA, On finite-difference approximations for normalized Bellman equations, to appear in Appl. Math. Optim.

[17] E.R. JAKOBSEn, On the rate of convergence of approximation schemes for Bellman equations, Math. Models Mehods Appl. Sci., 13:5 (2003), pp. 87-102.

[18] F. John, On integration of parabolic equations by difference methods I., Comm. Pure Appl. Math., 5 (1952), pp. 155-211.

[19] H.O. Kreiss, Über die Lösung des Cauchyproblems für lineare partielle Differenzialgleichungen mit Hilfe von Differenzengleichungen, Acta Math., 101 (1959), pp. 179-199.

[20] N.V. Krylov, On the rate of convergence of finite-difference approximations for Bellman's equation, St. Petersburg Math. J., 9:3 (1997), pp. 245-256.

[21] N.V. KRYlov, Approximating value functions for controlled degenerate diffusion processes by using piece-wise constant policies, Electronic Journal of Probability, 4:2 (1999), pp. 1-19.

[22] N.V. KRYLOv, The rate of convergence of finite-difference approximations for Bellman equations with Lipschitz coefficients, Appl. Math. and Optimiz., 52:3 (2005), pp. 365-399.

[23] N.V. KRYLOV, The rate of convergence of finite-difference approximations for Bellman equations with Lipschitz coefficients, Appl. Math. Optim., 52:3 (2005), pp. 365-399.

[24] N.V. KRYLOV, A priori estimates of smoothness of solutions to difference Bellman's equations with linear and quasilinear operators, Math. Comp., 76 (2007), pp. 669-698.

[25] O.A. LAdyzhenskaya, Boundary value problems of mathematical physics, Izdat. "Nauka", Moscow, 1973.

[26] S. Larsson and V. Thomée, Partial differential equations with numerical methods, texts in Applied Mathematics, 45. Springer-Verlag, Berlin, 2003.

[27] P.D. LAx, Numerical solution of partial differential equations, Amer. Math. Monthly, 72:2 (1965), part II, pp. 74-84.

[28] W. Littman, Résolution du problème de Dirichlet par la méthode des différences finies, C. R. 
Acad. Sci. Paris, 247 (1958), pp. 2270-2272.

[29] L.A. LyUsternik, A finite-difference analog of Green's function in the three-dimensional case, Vyčisl. Mat., 1 (1957), pp. 3-22 (in Russian).

[30] V.I. Mazhukin, D.A. Malafeit, P.P. Matus, and A.A. Samarskit, Difference schemes on nonuniform grids for equations of mathematical physics with variable coefficients, (in Russian) Zh. Vychisl. Mat. Mat. Fiz., 41:3 (2001), pp. 407-419; translation in Comput. Math. Math. Phys., 41:3 (2001), pp. 379-391.

[31] A.R. Mitchell and D.F. Griffith, The Finite Difference Methods in Partial Differential Equations, Wiley, London 1980.

[32] J. von Neumann and R. D. Richtmyer, A method for the numerical calculation of hydrodynamic shocks, J. Appl. Phys., 21 (1950), pp. 232-237.

[33] I.G. PetrovskiI, New proof of the existence of a solution of Dirichlet's problem by the method of finite differences, Uspekhi Matem. Nauk, 8 (1941), pp. 161-170 (in Russian).

[34] R.D. Richtmyer and K.W. Morton, Difference Methods for Initial-Value Problems, Interscience, New York 1967.

[35] A.A. SamarskiI, The theory of difference schemes, Monographs and Textbooks in Pure and Applied Mathematics, 240. Marcel Dekker, Inc., New York, 2001.

[36] G. Strang, On the order of convergence of the Crank-Nicolson procedure, J. Math. and Phys., 38 (1959/1960), pp. 141-144.

[37] J.C. Strikwerda, Finite Difference Schemes and Partial Differential Equations, Pacific Grove, CA: Wadsworth and Brooks, 1989.

[38] V. ThомéE, From finite differences to finite elements. A short history of numerical analysis of partial differential equations, Numerical analysis 2000, Vol. VII, Partial differential equations, J. Comput. Appl. Math., 128:1-2 (2001), pp. 1-54.

[39] V. Thомée, Finite Difference Methods for Linear Parabolic Equations, in Handbook of Numerical Analysis, Volume I, 3-196, (General Editors: P.G. Ciarlet and J.L. Lions) NorthHolland, Amsterdam-New York-Oxford-Tokyo 1990.

[40] A.N. Tikhonov and A.A. Samarskil, Homogeneous difference schemes, Zh. Vychisl. Mat. i Mat. Fiz., 1 (1961), pp. 5-63 (in Russian), U.S.S.R. Comput. Math. and Math. Phys., 1 (1961), pp. 5-67.

[41] O.B. WidLund, On the rate of convergence for parabolic difference schemes II, Comm. Pure Appl. Math., 23 (1970), pp. 79-96. 
